Aerospace Science and Technology, Volume 80, Issue September, 2018, pp. 587-603

DOI:10.1016/j.ast.2018.07.033

\title{
Effect of Design Parameters on the Mass of a Variable-span Morphing Wing Based on Finite Element Structural Analysis and Optimization
}

\author{
Pedro D.R. Santos ${ }^{\mathrm{a}, *}$, Diogo B. Sousa ${ }^{\mathrm{a}}$, Pedro V. Gamboa ${ }^{\mathrm{a}}$, Yifan Zhao ${ }^{\mathrm{b}}$ \\ ${ }^{a}$ Department of Aerospace Sciences, Faculty of Engineering, University of Beira Interior, Portugal \\ ${ }^{b}$ Through life Engineering Services Centre, Cranfield University, United Kingdom
}

\begin{abstract}
In the past years, the development of morphing wing technologies has received a great deal of interest from the scientific community. These technologies potentially enable an increase in aircraft efficiency by changing the wing shape, thus allowing the aircraft to fly near its optimal performance point at different flight conditions. However, these technologies often present an undesired mass increase due to their inherent complexity. Therefore, the aim of the current work is to ascertain the influence of geometrical and inertial parameters on the structural mass of a Variablespan Wing (VSW). The structural mass prediction is based on a parametric study. A minimum mass optimization problem with stiffness and strength constraints is implemented and solved, being the design variables structural thicknesses and widths, using a parametric Finite Element Model (FEM) of the wing. The study is done for a conventional fixed wing and the VSW, which are then combined to ascertain the VSW mass increment, i.e., the mass penalization of the adopted morphing concept. Polynomials are found to produce good approximations of the wing mass. The effects of the various VSW design parameters in the structural mass are discussed. On one hand, it was found that the span and chord have the highest impact in the wing mass. On the other hand, the VSW to fixed wing mass ratio proved that the influence of span variation ratio in the wing mass is not trivial. It is found that the mass increase does not grow proportionally with span variation ratio increase and that for each combination of span and chord, exists a span variation ratio that minimizes the mass penalty. In the future, the developed polynomials could be used to create a mass
\end{abstract}

\footnotetext{
${ }^{*}$ Corresponding author at: Department of Aerospace Sciences, Faculty of Engineering, University of Beira Interior, Portugal

Email address: p_daniel_santos@hotmail.com (Pedro D.R. Santos)
} 
prediction model to aid the design of morphing wings during the conceptual design phase.

Keywords: Morphing Wing, Variable-span Wing, Telescopic Wing, Optimization, Finite Element Model, Parametric Study, Composite Material Structure, Multivariable Polynomial Fitting

\section{Introduction}

Mass control, namely the process by which the lightest possible aeroplane is derived within the constraints of the design criteria [1], is an essential part of the design process of any aerospace vehicle. Accurate estimations of aircraft mass are vital in the early stages of an aircraft design process. They drive all the major choices in configuration and layout as well as being the main foundation of performance predictions. Mass estimation in aircraft design is very challenging due to the high number of variables involved in the creation of an accurate mass model, the numerous relationships between them and the high degree of uncertainty associated with the problem itself.

Morphing wing technologies require the concurrent development of design and optimization strategies to expedite overall development of these systems. The development of robust morphing wing sizing codes to be used during conceptual design tasks is of major importance, since it enables studies of the operational benefits and provide a methodological basis for future morphing aircraft sizing codes. However, such tools need accurate mass predictions for the major components, including the morphing wings. Therefore, morphing technologies will only be considered in new aircraft development, if mass predictions with sufficient accuracy are available. Simple and sufficiently accurate mass prediction methods for designing morphing wings at the conceptual design phases are rare. Therefore, the benefits that one morphing strategy can offer over another or even over a conventional fixed wing are thus quite difficult to assess without resorting to detailed time consuming Finite Element Model (FEM), normally only performed at the detailed design phase.

Most of the existing mass estimation models available in the literature can be classified into two main categories: semi-empirical and finite element [2]. Semi-empirical models are based on data from similar existing aircraft. Therefore, the robustness 
of these models depends on the similarities: size, configuration, and technology (systems, structural efficiency, and materials), between the aircraft under study and the aircraft that have been used in the derivation process of these models [3]. In the case of morphing wings, little information is currently available to substantiate such a wing mass prediction and thus, the sizing results would be unfounded. On the other hand, FEMs are not suitable to be used during the conceptual and preliminary design phases, since they require detailed knowledge of the internal geometry and aerodynamics that are usually not available early in the design process [4]. Thus, it is desirable to formulate a model based on FEM analyses, to capture the structural trends, but without the complexity posed by these methods.

Various studies have been performed in mass models for conceptual design of conventional wings. Some developed wing mass estimation models for commercial and transport aircraft $[2,5]$ and others for more unconventional configurations, such as nonplannar configurations $[6,3,7]$ or high speed transport [8]. However, very few studies have focused on morphing wings.

Frommer and Crossley [9] used a technique called photo-morphing. In this technique, the reference wing mass is computed using a mass model developed for conventional wings, the so called basic mass. Then, this value is corrected for actuation system mass, using the maximum variation in unique planform area, multiplied by an actuator specific (in relation to area) mass constant. This metric is indicative of the wing planform that needs to be moved when changing from one shape to another. Although the photo-morphing is simple to implement, it is very limiting, since there may be no skin materials or actuation strategy to achieve some of the represented shape changes. Additionally, the actuator specific mass constant is difficult or even impossible to derive for some morphing systems.

Probably, the most significant study was performed by Skillen and Crossley $[10,11]$, where they developed a wing mass model, considering the variation of span, chord and sweep. FEM analyses, based on equivalent box-beam models or shell type structures, were used to estimate the mass for a sufficient number of combinations and then a least square regression was used to approximate the wing mass. Validation examples were provided for chord and variable sweep morphing wings. No details about the span variation methodology were given. The shape variation con- 
sidered clearly required the use of high strainable skins, but they were not formally addressed in the FEM, potently resulting in erroneous mass estimations. Moreover, the actuation system was modelled using simplified hydraulic actuators, not being considered other types of actuators that could yield better results, specially for small sized Remotely Piloted Aircraft Systems (RPAS).

From what has been presented, methods to predict wing mass of morphing wings are limited and scarce. Morphing wings can have an undesired mass increase due to their inherent complexity both in the load carrying structure and in the actuation systems. This can potentially limit or even negate any performance benefits, depending on the intended flight mission and/or aeroplane type. However, mass estimation of morphing wings is difficult and very little is known about the impact of wingspan or wingspan maximum variation, among others, on the mass of the load carrying structure. Therefore, the present work has two main goals:

- Ascertain the influence of geometrical and inertial parameters on the structural mass of a Variable-span Wing (VSW) concept with an integrated Trailing Edge (TE) device;

- Develop mass prediction functions by fitting multivariable polynomial approximations. These can be used, in the future, to create a mass prediction model of VSWs that could be used during the conceptual design phases.

A total of five parameters were considered, namely, wingspan, wing chord, span variation ratio, flap chord ratio and aeroplane weight. To achieve this, a minimum mass optimization problem with stiffness and strength constraints was implemented and solved for a sufficient number of combinations of the wing parameters, being the design variables, structural thicknesses and widths. A parametric structural FEM of the wing was built in APDL and solved in ANSYS ${ }^{\circledR}$. Concurrently, the same study was performed for a conventional fixed wing. Using the computed data, mass and mass ratio functions were created by fitting multivariable polynomials: fixed wing mass, VSW mass and VSW to fixed wing mass ratio. The latter was used to ascertain the mass penalty associated with the adopted morphing concept. Additionally, the effects of various VSW design parameters in the structural mass were inferred and synthesized. 


\section{Variable-span Wing Concept}

\subsection{Wing Concept}

The morphing wing herein presented relies on a telescopic wing. The layout of the VSW concept is based on a hollow Inboard Fixed Wing (IFW) that is attached to the fuselage, inside of which an Outboard Moving Wing (OMW) slides actuated by an electromechanical mechanism. This concept consists of a two element rectangular telescopic wing containing a variable camber TE that starts next to the fuselage and extends in the spanwise direction up to the region where the moving element of the wing retracts into. The VSW does not possess ailerons, allowing for structural simplicity and improved aerodynamic performance. Rolling moments could be effectively controlled by asymmetrical wingspan variation.

Figure 1 shows a planform conceptual view of the VSW, where the main planform parameter names are identified for easier description. The VSW has some differenti-

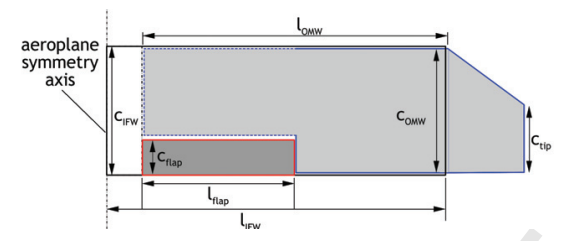

(a)

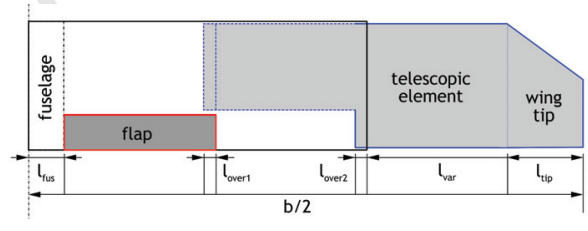

(b)

Figure 1: VSW conceptual planform view: (a) fully retracted and (b) fully extended configuration, with geometrical parameters identified.

ating factors, namely the use of a canted wingtip and a morphing flap. The wingtip is intended to provide additional lateral-directional stability. In fact, it creates an effective dihedral angle, without changing the telescopic sections of the wing, thus, decreasing the structural and actuation system complexity that would result from non-flat wing sections. Additionally, the wingtip increases the overall aerodynamic efficiency. Finally, the addition of the morphing flap enables camber changes, resulting in an increase in lift-to-drag ratio at different flight lift coefficients.

Both IFW and OMW wing panels have the design constraint of keeping chord and aerofoil geometry constants along each panel span, enabling proper fitting and support of the OMW. Observing Fig.1 one can see that the IFW length is denoted by $l_{I F W}$, the OMW length by $l_{O M W}$, the tip length by $l_{t i p}$ and the flap length by $l_{\text {flap }}$. The $l_{\text {var }}$ parameter refers to the span length that is variable as a result of the 
movement of the OMW. There are two regions of contact that aid the load transfer from the OMW to the IFW: $l_{\text {over } 1}$ and $l_{\text {over } 2}$. The former is the innermost region and is responsible for carrying the majority of the bending and torsional moments of the OMW. The latter corresponds to the outermost contact region, being responsible for providing stability in the chordwise direction. There is an additional length parameter that takes into account the fuselage length, called $l_{f u s}$. Finally, IFW, OMW, flap and tip chords are designated as $c_{I F W}, c_{O M W}, c_{f l a p}$ and $c_{t i p}$, respectively.

The shape and size of the VSW reference design was obtained through an inhouse computational constrained aerodynamic shape optimization code, aimed at determining the wing mean chord and span values that minimize its drag for the specified mission profile. A detailed description of the aerodynamic optimization procedure is given in [12]. Along with the optimization procedure, two geometrically compatible aerofoils were provided in a way that the OMW slides inside the IFW. The method employed to geometrically offset the aerofoils and determine its aerodynamic performance is fully explained in [13].

\subsection{Structural Concept and Materials}

In principle, morphing wings tend to be heavier than conventional fixed wings due to increased structural and actuation complexity. Therefore, the structure should be designed and materials selected in such a way that sufficiently light wing components are attained. This facilitates integration into a realistic application while maintaining rigidity to carry flight loads. Therefore, the VSW uses a semi-monocoque structural concept, which consists in a stressed skin construction that carries shear loads, reinforced by multiple spars to carry bending and torsion moments.

Figure 2 shows a schematic of the IFW and OMW wing cross-sections, where the main sectional parameters are identified to facilitate the description.

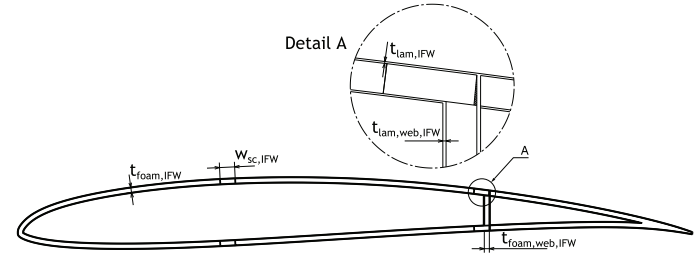

(a)

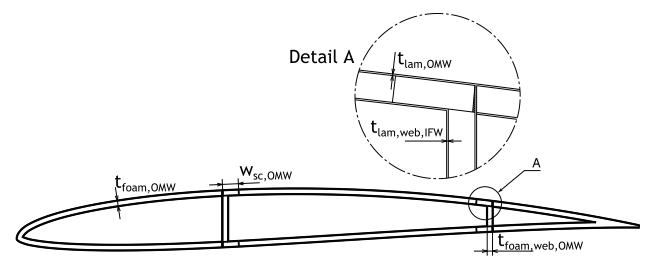

(b)

Figure 2: VSW cross-section view with sectional parameters identified: (a) inboard fixed wing and (b) outboard moving wing sections. 
Observing Fig.2, it is visible that both sections are composed by a composite sandwich made up with a foam core and two plies of bidirectional laminated carbonepoxy composite. The fibres of the laminate are orientated at an angle of $\pm 45^{\circ}$. The four unidirectional pultruded carbon-epoxy composite spar caps are integrated into the skin, two in the upper surface and the other two in the lower surface. The two frontal spar caps are located in the thicker aerofoil section (30\% of the local chord), while the two rear spar caps are located at a chord ratio dictated by the flap chord $\left(c_{\text {flap }}\right)$.

Looking at the IFW section (Fig.2(a)), only one web (vertical element of the wingbox) is visible and it does not extend the full length. Instead, it is interrupted at the end of the flap position $\left(l_{\text {fus }}+l_{\text {flap }}\right)$ from its root, in order to allow the OMW to retract into the IFW. Due to this constraint, no internal ribs can be used. Therefore, chordwise reinforcements made with unidirectional laminated carbon-epoxy composite are applied in three critical sections: beginning/end of the flap and IFW tip.

In Fig.2(b) it can be seen that the OMW has two webs. They extend from the component's root to the end of the rectangular portion (beginning of wing tip). Similar to the IFW, the two frontal spar caps and webs are located in the thicker aerofoil section (30\% of the local chord), while the two rear spar caps and webs are located in a chord ratio dictated by the flap chord $\left(c_{\text {flap }}\right)$. There are no chordwise reinforcements in the OMW, since four internal ribs are used: OMW root and $l_{\text {over } 2}$ location, end of the rectangular portion and end of wingtip.

Several sectional parameters are identified in Fig.2: $t_{l a m}$ and $t_{l a m, w e b}$ denote the laminate thicknesses of the skin contour and web; $t_{\text {foam }}$ and $t_{\text {foam,web }}$ denote the foam core thicknesses of the skin contour and web; and $w_{s c}$ denotes the width of the spar caps. The subscripts IFW and OMW are added to the mentioned terminology, in order to differentiate the parameters of the former and latter sections.

The VSW is made with three materials: carbon-fibre fabric with epoxy for the faces of the sandwich; PVC foam (Airex ${ }^{\circledR}$ C70.90) for the core of the sandwich and internal ribs; and pultruded unidirectional carbon-fibre with epoxy for the spar caps (vDijk high-strength pultrusions).

Material properties of the PVC foam and pultruded unidirectional carbon-fibre were obtained from the manufacturer's datasheet. The properties of the sandwich 
faces are assumed for a hand lay-up procedure with vacuum curing [14].

The properties of these materials are presented in Table 1. The pultruded carbon fibre and woven carbon/epoxy are formulated as orthotropic materials and the PVC foam is considered to be an isotropic material. Note that subscripts 1 and 2 denote the direction of the fibre and perpendicular to the fibre, respectively (where applicable).

Table 1: Material properties used in the VSW parametric study.

\begin{tabular}{cccc}
\hline Property & $\begin{array}{c}\text { Woven } \\
\text { carbon/epoxy }\end{array}$ & $\begin{array}{c}\text { Pultruded } \\
\text { carbon/epoxy }\end{array}$ & Airex ${ }^{\circledR}$ C70.90 \\
\hline$\rho, \mathrm{kg} / \mathrm{m}^{3}$ & 1500 & 1600 & 100 \\
$E_{1}, \mathrm{GPa}$ & 46 & 105 & 0.084 \\
$E_{2}, \mathrm{GPa}$ & 46 & 7.5 & - \\
$G_{12}, \mathrm{GPa}$ & 3.25 & 3.75 & 0.040 \\
$\nu_{12}$ & 0.1 & 0.3 & 0.05 \\
$F_{t u 1}, \mathrm{MPa}$ & 600 & 1500 & 2.7 \\
$F_{t u 2}, \mathrm{MPa}$ & 600 & 50 & - \\
$F_{c u 1}, \mathrm{MPa}$ & 570 & 1200 & 2 \\
$F_{c u 2}, \mathrm{MPa}$ & 570 & 250 & - \\
$S_{12}, \mathrm{MPa}$ & 90 & 70 & 1.7 \\
\hline
\end{tabular}

\section{Parametric Study Methodology}

The most common approach to developing fixed-wing mass predictions is centred on the idea that a large database of wing masses and their associated geometry already exists through previous developed aircraft. Direct application of the above approach is not possible for morphing wing components because an adequate set of aircraft data does not exist. The approach used here is to develop a wing mass database and develop an equation that approximates this database. Using a Design of Experiments (DOE) to define a set of morphing wings with various shapes, representative FEMs were developed for each wing in the database and then sized to give a corresponding mass estimate. These data can then be approximated using an appropriate basis equation using a least squares regression technique resulting in the morphing wing mass equation. Only the structural mass was considered here. The various aspects of this procedure are described in detail in the following sections.

The VSW presented in detail in the previous section serves as a basis to perform the parametric study with a double purpose. On one hand, it allows the study of the influence of a set of geometrical and inertial parameters on the structural mass 
of the VSW. On the other hand, as already mentioned, it allows the creation of the mass database, needed to create the approximation to predict the structural mass of the VSW. A total of five parameters were selected to perform the study. These are

1. $b$ - wingspan in fully extended configuration;

2. $c_{I F W}$ - inboard fixed wing chord;

3. $\bar{l}_{\text {var }}$ - wing variable-span (VS) ratio (with respect to semi-span);

4. $\bar{c}_{\text {flap }}$ - flap chord ratio (with respect to $c_{I F W}$ );

5. W - aeroplane Maximum Takeoff Weight (MTOW).

The first four parameters are called the wing geometrical base parameters, since all the other wing dimensions are derived from those. Parameters one and two are illustrated in Fig.1. Parameters three and four, $\bar{l}_{\text {var }}$ and $\bar{c}_{\text {flap }}$, are nondimensionalized versions of $l_{\text {var }}$ and $c_{\text {flap }}$, using the semi-span and the IFW chord, respectively. The fifth mentioned parameter, the aeroplane MTOW, $W$, was included in order to size the wings with different loading conditions and is used to compute the maximum load factor, using the CS-VLA regulation (described in Section 3.4).

For each set of parameters a minimum mass optimization problem with stiffness and strength constraints was implemented and solved, being the design variables structural thicknesses and widths. The optimization and structural FEM of the wing were developed in ANSYS ${ }^{\circledR}$ APDL.

The characteristics of an in-house developed RPAS were used to define a reference wing configuration. Those characteristics are: MTOW of $150 \mathrm{~N}$; IFW chord of $0.257 \mathrm{~m}$; span of $3.554 \mathrm{~m}$ in the fully extended configuration and a span of $3.104 \mathrm{~m}$ in the fully retracted configuration, giving a span variation to span ratio of 0.25 .

\subsection{Optimization}

The optimization was carried out for each wing configuration, with the purpose of minimizing wing mass and, at the same time, ensure that each wing supports the prescribed loading. ANSYS ${ }^{\circledR}$ Mechanical APDL internal optimization facilities were used to carry out the optimization. The first order method was used, since it is the most accurate method available [15]. This method of optimization computes and uses derivative information. The constrained problem statement is transformed into 
an unconstrained one via penalty functions. Derivatives are formed for the objective function and the state variable penalty functions, using central finite differences, leading to a search direction in the design space. Various steepest descent and conjugate direction searches are performed during each iteration until convergence is reached. Each iteration is composed of multiple sub-iterations that include search direction and gradient computations [15].

The Design Variables (DV) adopted in the current study were: IFW and OMW laminate thicknesses, $t_{l a m, I F W}$ and $t_{l a m, O M W}$, and IFW and OMW spar cap widths, $w_{s c, I F W}$ and $w_{s c, O M W}$ (see Fig.2). Maximum bounds of laminate thicknesses were chosen based on the limitation of the thin shell element derivation assumptions, since shells elements can not have a radius of curvature to thickness ratio $\leq 0.5$. Notice that these upper bounds are only used to improve optimization stability and they do not have an impact on the final optimized values. The maximum spar cap widths were dictated by geometric constraints, i.e., maximum width that avoids mutual intersection. In the IFW, this is a function of the local and flap chord. In the OMW, it is only a function of the local chord.

Three constraint functions were adopted: maximum tip deflection and rotation, and ratio of elements that display failure. The tip deflection, $w_{t i p}$, was limited to $2.5 \%$ of the span $(b)$ and the tip rotation, $\theta_{t i p}$, to be between -0.6 and $0.6^{\circ}$. These constraints were necessary to allow an even slide of the two wing components. In fact, if the tip displacement or rotation were too large, the VSW mechanism could eventually jam, compromising system integrity and functionality. These bounds were derived from past experience [14]. Additionally, a failure criterion constraint was used to detect structural failure of the wing. The inverse of Tsai-Wu strength ratio index failure criterion was used. In the current implementation, the ratio of the failed elements, $\overline{S R}_{T W}$, was imposed to be less than $0.1 \%$ of the total number of elements, rather than imposing that all elements do not display failure. This is due to the possibility of existing small areas with failed elements, that could be easily solved using local reinforcements. Since the current study is appropriate for the conceptual design phases, these small areas should not drive the overall design. 
In short, the optimization problem can be written as

$$
\begin{aligned}
& \text { Minimize: } m_{\text {wing }}=f\left(w_{s c, I F W}, t_{\text {lam }, I F W}\right. \text {, } \\
& \left.w_{s c, O M W}, t_{l a m, O M W}\right) \\
& \text { Subjected to: } w_{s c, I F W, \min }<w_{s c, I F W}<w_{s c, I F W, \max } \\
& w_{s c, O M W, \min }<w_{s c, O M W}<w_{s c, O M W, \max } \\
& t_{\text {lam,IFW,min }}<t_{\text {lam,IFW }}<t_{\text {lam,IFW,max }} \\
& t_{\text {lam,OMW,min }}<t_{\text {lam,OMW }}<t_{\text {lam,OMW,max }} \\
& w_{t i p}<0.025 b \\
& \left|\theta_{\text {tip }}\right|<0.6^{\circ} \\
& \overline{S R}_{T W}<0.001
\end{aligned}
$$

Figure 3 summarizes the general flow of steps in the developed parametric design script.

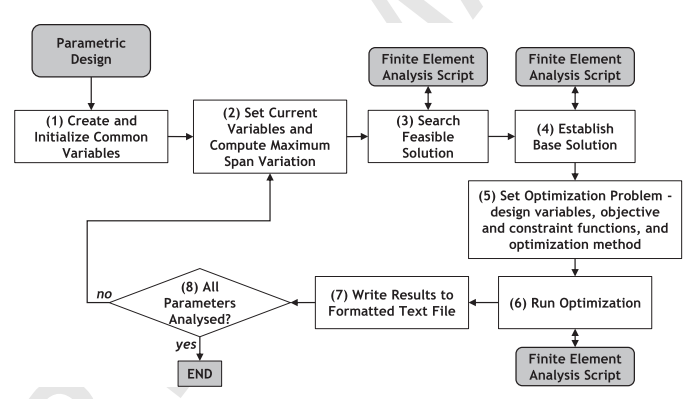

Figure 3: Parametric design script flowchart.

Observing Fig.3, one can divide the work-flow in the following steps:

1. Variable initialization: variables are initialize using the user defined parameters (geometrical base parameters, aeroplane MTOWs, constraint function limits, among others).

2. Parameters calculation: Step 2 signals the start of the DOE loop. Design dependent parameters are calculated using equations defined in the geometric scaling (described in Section 3.5). Additionally, maximum span variation for the configuration under evaluation is computed.

3. Search feasible solution: This step is critical to enhance the stability and convergence time of the optimization procedure. The parametric study base variables and a first guess of the design variables are used to evaluate the feasibility of the 
wing structure. A solution is considered feasible if it meets all the constraint functions. Wing deformations and stresses are computed using the FEM script (subject of the next section). This first guess is a user prescribed value if it is the first run or the previous optimized values if otherwise. Two loops are used: a coarser loop to quickly find design values that satisfy the constraints and a finer loop to refine these values. In the coarser loop a multiplier is used, which starts at 1 and increases by 1 each loop, changing the values of the design variables until a feasible solution is found. In the finer loop, the same technique is used 8 times with decreasing multipliers $(0.5,0.25,0.125 \ldots)$.

4. Establish base solution: The optimization procedure requires a starting solution to be performed. This solution is done using the design variables determined in the previous step.

5. Optimization initialization: Here the optimization problem is defined, namely design variables, objective and constraint functions, and optimization method.

6. Optimization: In step 6, ANSYS ${ }^{\circledR}$ internal optimization facilities are started. The optimization continues until convergence is achieved by comparing the current iteration design set to the previous set and the best set, using an objective function tolerance (chosen by the user), or until the maximum number of iterations is reached [15].

7. Write results: The main outputs of the optimization are written, namely: final values of the optimization variables, tip deflection and rotation, fraction of elements that display failure, among others.

8. DOE check: Evaluate if all the values in the DOE were already analyzed. If the check is true the loop ends. If the check is false the script continues to step 2 and proceeds to the evaluation of the next wing configuration.

\subsection{Finite Element Analysis}

The numerical model of the VSW wing was developed using the ANSYS ${ }^{\circledR}$ Parametric Design Language (APDL) [16] with shell elements according to the base geometrical variables and design variables. The APDL script handles geometry creation, material definition, section properties, meshing, analysis and post-processing. The developed script allows the computation of a static solution (deformations, rotations 
and stresses) using small deformations. Due to computational resource optimization, SHELL281 element was used to discretize the surfaces. This element is an eight-node element suitable for analysing thin to moderately-thick shell structures. It has a total of six degrees of freedom at each node: translations in the $x, y$ and $z$ directions, and rotations about the $x, y$ and $z$-axes. The element is well-suited for linear, large rotation, and/or large strain nonlinear applications.

The IFW sandwich skin was modelled with three layers built as offset surfaces from the aerofoil contour according to their own thickness. These three layers constitute the carbon/epoxy faces and foam core. In the locations of the embedded spar, the foam layer was replaced with rectangular cross-section unidirectional pultruded carbon/epoxy rods. Likewise, the OMW was discretized using the same approach.

The peculiar structure used by the VSW, required the use of contact elements, in order to correctly model the interface. The contact in the overlapping surfaces between the IFW and the OMW was modelled with a shell to shell contact using TARGE170 (target element for 3D geometries surfaces) and CONTA174 (contact element for $3 \mathrm{D}$ shells with mid side nodes). The contact elements were added to the $l_{\text {over } 1}$ and $l_{\text {over } 2}$ regions. In order to reduce computational cost, an asymmetric contact was created. In this type of contact, one surface is designated to be the target and the other a contact surface. Then, one contact pair is created between surfaces. Contrary, in the symmetric contact both surfaces are designated as target and contact, which requires the creation of two contact pairs. Consequently, the asymmetric contact is more efficient than the symmetric contact. The contact elements' behaviour was chosen to be bonded, in order to reduce the computation cost of each analysis. However, an increase in the local stiffness is to be expected, which has the effect of underestimating the wing deformations. The bonded contact uses a Multipoint Constraint (MPC) formulation. MPC uses rigid constraint equations between the elements on the contact and target faces to model the bonded connection. The connection locations are determined using the contact element pinball radius and then the contact elements are replaced with internal constraint equations.

The performed solution was a static analysis, without large deflections and using the MPC based contact interface. Given these characteristics, the solution was linear, greatly reducing the computation time for each static analysis. In turn, this 
improved the robustness of the optimization, since it reduces the possibility of unconverged solutions. It should be added that since a symmetric wing planform was assumed, only one wing was modelled. Figure 4 summarizes the general structure of the developed FEM script.

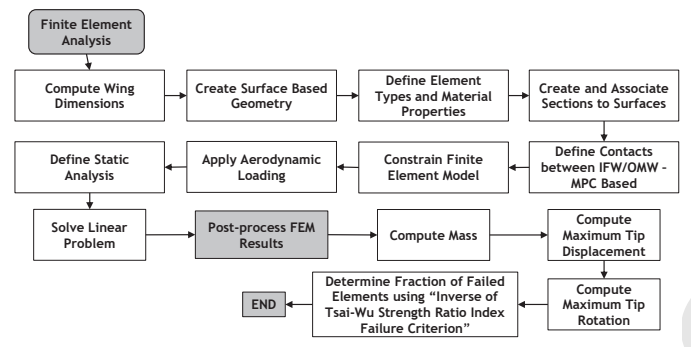

Figure 4: Finite element model flowchart.

\subsection{Polynomial Fitting}

Several basis functions can be used to approximate the mass of the wings under study. In the present study, a full quadratic polynomial was used. The general form of such equation is given by

$$
m\left(\left\{X_{1: n}\right\}\right)=a_{0}+\sum_{i=1}^{n} a_{i} X_{i}+\sum_{i=1}^{n} \sum_{j=i}^{n} a_{i j} X_{i} X_{j}
$$

where, $\left\{X_{i}\right\}$ represents one of the $n$ parameters within the DOE and $a_{i j}$ represent the unknown coefficients in each of the mass equations. The quadratic approximation captures both linear and quadratic terms and tends to provide a good approximation when the design set displays moderate deviations from linear behaviour. Therefore, it can approximate the wing mass if a non-linear relationship of the wing mass parameters is verified. The second order polynomial allows a better study of the effect on the wing mass produced by each individual parameter, since both linear and two level interaction relationships between parameters could be identified.

A total of two structural mass and one mass ratio equations were developed: fixed wing mass, VSW mass and VSW to fixed wing mass ratio. Multivariate polynomials were then created, $m_{s t r, f w}, m_{s t r, V S W}$ and $\bar{m}_{s t r, V S W / \text { fixed }}$ being a function of the wing 
parameters in the following form

$$
\begin{aligned}
m_{\text {str }, f w} & =f\left(b, c_{I F W}, \bar{c}_{\text {flap }}, W\right) \\
m_{\text {str }, V S W} & =f\left(b, c_{I F W}, \bar{l}_{\text {var }}, \bar{c}_{\text {flap }}, W\right) \\
\bar{m}_{\text {str }, V S W / f w} & =f\left(b, c_{I F W}, \bar{l}_{\text {var }}, \bar{c}_{\text {flap }}, W\right)
\end{aligned}
$$

where, Eqs.(3), (4) and (5) are functions of the already mentioned parametric study parameters, with the exception of the first (fixed wing polynomial) that does not have VS ratio. Notice that Eq.(5) is not a mass equation since it represents the ratio of VSW mass to fixed wing mass, thus being a non-dimensional quantity.

A mathematical algorithm was applied to find the unknown polynomial coefficients. The used method is based on an extension to the Granger causality test, called ERR-causality [17]. The main advantage of the ERR test is that it can be applied to nonlinear multivariate systems. Furthermore, with the ERR-causality method it is possible to organize the polynomial terms, in respect to its significance to the overall solution. Therefore, the complete polynomial model does not have to be used to provide a good polynomial approximation. The minimum number of significant terms that adequately fits the dataset is evaluated by calculating the Sum of Error Reduction Ratio (SERR) values. Using this metric, the final simplified polynomial can be obtained, by screening the terms that have lower influence in the dependent variable and eliminating them.

The resulting polynomials were evaluated using goodness of fitting parameters: coefficient of determination, $R^{2}$, maximum relative error, maximum absolute error and Root Mean Squared Error (RMSE). The $R^{2}$ correlates the computed data with the predicted data by a square of the sample correlation coefficient, where values near to one indicate a good fit. RMSE is a fit standard error for regression and estimates the standard deviation of each data component [18].

After computing the polynomial, it was important to visually validate the results by plotting the representation of the resulting polynomials with the data points overlaid for comparison reasons. These plots facilitated final considerations to be taken about the trends of each parameter on the wing mass. The five independent variables describe a hypersurface with a six-dimensional representation. As this is 
not possible to plot, the complete polynomial approximations were reduced to several polynomials with only two independent variables.

\subsection{Loading}

The design load cases are important to determine realistic sizes of the wing's structural components. These load cases are usually defined by the limits of the V-n diagram. In fact there may be combinations of bending and torsion moments outside the corner points which may size individual components. Ideally, the wing structure would be optimized with respect to all expected loading and operating conditions. However, this results in an extremely large number of loading conditions. Therefore, selecting only the critical loading condition should suffice to demonstrate the present approach.

In general terms, the morphing aircraft is expected to perform a long-endurance loiter, followed by a high speed dash. The morphing wing would have a high aspect ratio during loiter to reduce induced drag. During dash, the wing would have a low aspect ratio. Thus, the maximum speed of the dash wing is significantly higher than that of the loiter wing. Therefore, each configuration of the morphing wing has its own V-n diagram and, consequently, each configuration has its own set of design loads.

To extract the different design loads, the reference baseline VSW was analysed in three wing configurations: maximum, intermediate and minimum span. For each configuration, manoeuvre and gust V-n diagrams were computed, using the EASA's Certification Specifications for Very Light Aeroplanes, CS-VLA [19] and the RPAS specifications. From each V-n diagram the critical design points were extracted based on the maximum load factor which corresponds to the maximum bending moment.

For aeroplanes with low wing loading $(W / S)$, the gust envelope is critical [20]. Therefore, the maximum load factor of the gust diagram was used as design point. The next paragraphs explain in more detail the rational behind this methodology. Figure 5 shows a schematic of the positive portion of the V-n diagram, where the gust diagram is critical.

Observing Fig.5, three curves can be seen: cruise speed gust, dive speed gust and maximum lift coefficient. A total of four important speeds are also identified: design 


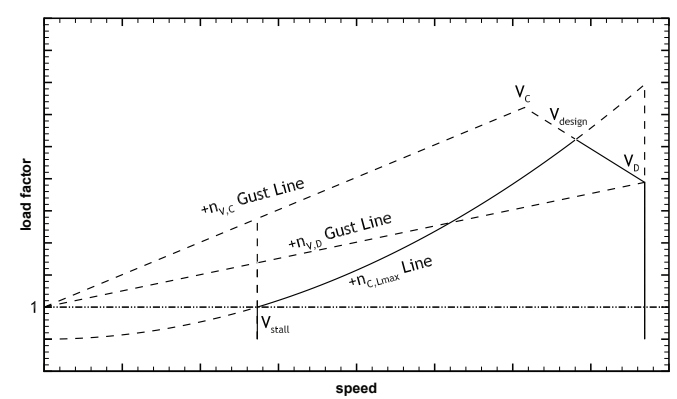

Figure 5: Schematic of the positive portion of the V-n diagram, where the gust diagram is critical.

cruise speed, $V_{C}$, design dive speed, $V_{D}$, stall speed, $V_{\text {stall }}$, and design speed, $V_{\text {design }}$. Referring to CS-VLA norm, the design cruise speed, $V_{C}$, and the dive speed, $V_{D}$, are computed using the following equations

$$
\begin{aligned}
& V_{C}=V_{C, \text { min }}=2.4 \sqrt{W / S} \\
& V_{D}=V_{D, \text { min }}=1.25 V_{C}
\end{aligned}
$$

being, $W$ the aircraft weight and $S$ the wing area. Analysing Eq.(6), one concludes that cruise speed is a function of the wing loading.

The cruise and dive gust curves are computed, as functions of speed, according to the CS-VLA norm, using the following equations

$$
\begin{aligned}
& n_{V_{C}}=1+\frac{\rho_{\text {air }} V C_{L_{\alpha}} K_{g} V_{g, C}}{2 W / S} \\
& n_{V_{D}}=1+\frac{\rho_{\text {air }} V C_{L_{\alpha}} K_{g} V_{g, D}}{2 W / S}
\end{aligned}
$$

where $K_{g}$ is the gust alleviation factor, $C_{L_{\alpha}}$ is the wing lift curve slope, $V_{g, C}$ and $V_{g, D}$ are the gust vertical speeds in cruise and dive conditions, respectively. Note that the gust vertical speeds are constant values (given in CS-VLA). The gust alleviation factor and the aeroplane mass ratio, $\mu_{g}$ are introduced to correct for dynamic effects in the aircraft pitching and vertical motion and the time lag during which lift is building. They are computed from

$$
K_{g}=0.88 \frac{\mu_{g}}{5.3+\mu_{g}} \quad \text { with } \quad \mu_{g}=\frac{2 W}{\rho_{a i r} c_{I F W} C_{L_{\alpha}} S}
$$

The lift curve slope is estimated from the aerofoil lift curve slope, considering an 
inviscid, incompressible flow over a wing with general planform [21]. Thus

$$
C_{L_{\alpha}}=\frac{C_{l_{\alpha}}}{1+C_{l_{\alpha}} /(\pi A R)(1+\tau)}
$$

where, $C_{l_{\alpha}}$ is the aerofoil lift curve slope, $A R$ is the wing aspect ratio and $\tau$ is a function of the wing planform. In the current work, although the wing planform is not elliptic, the lift distribution is assumed to have an elliptic shape. Therefore, $\tau$ can be neglected.

The stall load factor is computed using the definition of lift for maximum lift coefficient. Thus

$$
n_{C_{L, \max }}=\frac{0.5 \rho_{\text {air }} V^{2} S C_{L, \max }}{W}
$$

From Fig.5, one can see that, for low speeds, the $n_{C_{L, \text { max }}}$ curve is the limiting load factor. When the cruise speed is reached, the load factor reduces linearly between the two gust curves. The maximum gust load factor speed is found in the intersection between $n_{V_{C}}$ and $n_{V_{D}}$ curves and $n_{C_{L, \text { max }}}$ curves, denoted by $V_{\text {design }}$. Therefore

$$
n_{V_{C}}+\frac{n_{V_{D}}-n_{V_{C}}}{V_{D}-V_{C}}\left(V_{\text {design }}-V_{C}\right)=\frac{0.5 \rho_{\text {air }} V_{\text {design }}^{2} S C_{L, \text { max }}}{W}
$$

Solving Eq.(13) for $V_{\text {design }}$, yields the speed for the maximum gust load factor. The design load factor can now be determined by substituting the computed speed in the load factor for the maximum lift coefficient. Thus

$$
n_{\text {design }}=\frac{0.5 \rho_{\text {air }} V_{\text {design }}^{2} S C_{L, \max }}{W}
$$

After computation of the design load factor, the design lift force can be readily computed from

$$
L_{\text {design }}=n_{\text {design }} W
$$

Drag and pitching moment can now be computed. The drag was determined using the lift-to-drag ratio of the baseline wing. This quantity was corrected using the aspect ratio, if a different wing is considered. Therefore

$$
D_{\text {design }}=\frac{L_{\text {design }}}{(L / D)_{b l w} A R / A R_{b l w}}
$$


where, $(L / D)_{b l w}$ and $A R_{b l w}$ are the lift-to-drag ratio and aspect ratio of the baseline wing, respectively.

The pitching moment is computed from the baseline wing pitching moment coefficient and the design speed. Thus

$$
M_{\text {design }}=0.5 \rho_{\text {air }} V_{\text {design }}^{2} S c_{I F W} C_{M, b l w}
$$

being $C_{M, b l w}$ the pitching moment coefficient of the baseline wing. The pitching moment coefficient used is an average value in the studied range of lift coefficients of the baseline wing. It was assumed constant for all studied wing configurations.

The lift was considered to have an elliptic distribution, applied along the span at $25 \%$ chord position. Both drag and pitching moment distributions were assumed uniform along the wingspan. Lift and drag forces are perpendicular and parallel, respectively, to the free stream direction. However, since the effect of the AOA was not considered, they are assumed to be perpendicular and parallel to the wing chord line, respectively. Therefore, lift force is the vertical force, $F_{V}$, and the drag force is the horizontal force, $F_{H}$.

Finally, in order to better represent the load distributions along the chord, the initial force system of one vertical and horizontal force, applied at $25 \%$ of the wing chord, and a torsion moment about this same point, was substituted by two vertical forces applied at the fore and aft wing-box webs and four horizontal forces applied at each spar cap corner, as shown in Fig.6.

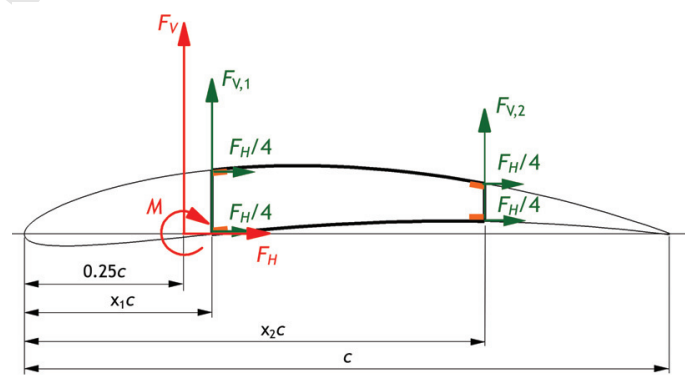

Figure 6: Equivalent force system of the VSW parametric study ( $F_{V}$ is the vertical force, $F_{H}$ the horizontal force and $M$ the pitching moment). 
From the force and moment equilibrium, forces $F_{V, 1}$ and $F_{V, 2}$ are found to be

$$
\begin{aligned}
& F_{V, 1}=F_{V}-\frac{M-0.25 c F_{V}+\bar{x}_{1} c F_{V}}{c\left(\bar{x}_{1}-\bar{x}_{2}\right)} \\
& F_{V, 2}=\frac{M-0.25 c F_{V}+\bar{x}_{1} c F_{V}}{c\left(\bar{x}_{1}-\bar{x}_{2}\right)}
\end{aligned}
$$

where, $\bar{x}_{1}$ and $\bar{x}_{2}$ are the ratios of the fore and aft spar positions to the chord length of the VSW. In the studied case, the fore ratio is 0.3 and the aft ratio is dependent on the flap chord $\left(1-\bar{c}_{\text {flap }}\right)$.

The fore vertical load was distributed along the frontal web of the wing, the aft vertical force was distributed along the rear web of the wing and the horizontal force was equally distributed by the four spar caps.

\subsection{Geometric Scaling}

In the parametric study, different wing geometric configurations were used. Thus, there was a need to create geometric mathematical relations between the different used parameters. In order to perform the scaling, a baseline or reference wing was used, being its dimensions designated using the subscript blw, where "bl" stands for baseline and "w" to wing. The geometric scaling can be divided into spanwise, chordwise and sectional parameters. As the names implies, the former denotes parameters in the spanwise direction and the second in the chordwise direction. The latter denotes mathematical relations of the section thicknesses and widths.

\subsubsection{Spanwise Formulas}

In the spanwise scaling, the important input parameters are the wingspan, $b$, and the VS ratio, $\bar{l}_{\text {var }}$. The innermost overlap between the IFW and OMW, $l_{\text {over } 1}$ is scaled using the OMW exposed area (moving part) and tip area, as well as the $y$ centroid of the same sections. In fact, with increasing $l_{v a r}$ the bending moment in the contact surface location increases and section centroid location moves away from the wing's root. Consequently, $l_{\text {over } 1}$ needs to increase to allow a smooth load transfer. The derived mathematical relation is given by

$$
l_{\text {over } 1}=\frac{l_{\text {over } 1, b l w}}{A_{\text {var }+ \text { tip }, \text { blw }} \bar{y}_{\text {var }+ \text { tip }, \text { blw }}} A_{\text {var }+ \text { tip }} \bar{y}_{\text {var }+ \text { tip }}
$$


where, $A_{\text {varttip }}$ is the area of the moving portion of the OMW plus the tip area and $\bar{y}_{\text {var+tip }}$ is the $y$ section centroid of the moving portion of the OMW plus the tip. Note that the VS length, $l_{v a r}$, is directly computed from the semi-span and VS ratio. Thus

$$
l_{\text {var }}=0.5 b \bar{l}_{v a r}
$$

The tip length, $l_{t i p}$ is computed using a linear scaling relative to the span. Therefore

$$
l_{t i p}=l_{t i p, b l w} \frac{b}{b_{b l w}}
$$

Finally, the IFW length, $l_{I F W}$, OMW length, $l_{O M W}$ and flap length, $l_{\text {flap }}$ can be readily computed by careful analysis of Fig.1. Thus

$$
\begin{aligned}
l_{I F W} & =0.5 b-l_{\text {var }}-l_{\text {tip }} \\
l_{\text {OMW }} & =2 l_{\text {var }}+l_{\text {over } 1}+l_{\text {over } 2} \\
l_{\text {flap }} & =l_{\text {var }}+l_{\text {over } 1}
\end{aligned}
$$

\subsubsection{Chordwise Formulas}

In the chordwise scaling, the fundamental parameter inputs are the IFW chord, $c_{I F W}$ and the flap chord ratio, $\bar{c}_{\text {flap }}$. The flap chord can be readily computed using the flap chord ratio and the IFW chord. Hence

$$
c_{\text {flap }}=\bar{c}_{\text {flap }} c_{I F W}
$$

Both OMW chord, $c_{O M W}$ and tip chord, $c_{t i p}$ are computed by linearly scaling the reference value relative to the IFW chord. Therefore

$$
\begin{aligned}
c_{O M W} & =c_{O M W, b l w} \frac{c_{I F W}}{c_{I F W, b l w}} \\
c_{t i p} & =c_{t i p, b l w} \frac{c_{I F W}}{c_{I F W, b l w}}
\end{aligned}
$$

The aerofoil geometry is fixed (section 2) and, consequently, curvature, aerofoil thickness-to-chord ratio and LE radius, are all constant in the studied wing configurations. 


\subsubsection{Cross-section Formulas}

IFW and OMW sections are composed of different structural elements, whose nomenclature as been previously elucidated in Fig.2 (section 2). All sectional parameters are either constant or derived from the wing design variables: laminate thicknesses and spar caps widths. IFW web laminate thickness, $t_{l a m, w e b, I F W}$, is considered to be twice the skin laminate thickness, $t_{\text {lam,IFW }}$, and the OMW web laminate thickness, $t_{l a m, w e b, O M W}$ is considered to be equal to $t_{l a m, O M W}$. Thus

$$
\begin{gathered}
t_{\text {lam,web }, I F W}=2 t_{\text {lam }, I F W} \\
t_{\text {lam }, w e b, O M W}=t_{\text {lam }, O M W}
\end{gathered}
$$

The IFW skin foam thickness, $t_{\text {foam }, I F W}$, and the web foam thickness, $t_{\text {foam, web }, I F W}$, does not change throughout the parametric study. The same is valid for the OMW sections $\left(t_{\text {foam, } O M W}\right.$ and $\left.t_{\text {foam,web,OMW }}\right)$.

\subsection{Fixed Wing Reference Design}

The fixed wing reference design was used to gauge the mass increase when considering the structural methodology of the VSW. In order to be comparable, the fixed wing has similar planform dimensions and cross-sections. However, the variable span ratio is zero, i.e., no outer moving wing. Due to the fact that the fixed wing was used as a reference, a more complex skin optimization layout was developed. In particular, the fixed wing skin was divided into four different regions, in order to allow the laminate thicknesses to vary along the span. However, the spar cap width was still kept constant in the spanwise direction, i.e., the spar caps remain rectangular. Therefore, a total of five design variables were used: four skin laminate thicknesses and one spar cap width. Figure 7 shows a model of the fixed wing, where the different optimization skin laminate areas were coloured to aid its identification.

\section{Parametric Study Results}

As presented in previous sections, the chosen design parameters are: wingspan, $b$, IFW wing chord, $c_{I F W}$, VS ratio, $\bar{l}_{v a r}$, flap chord ratio, $\bar{c}_{\text {flap }}$, and aeroplane MTOW, $W$. For each input parameter the selected values are presented in Table 2. 


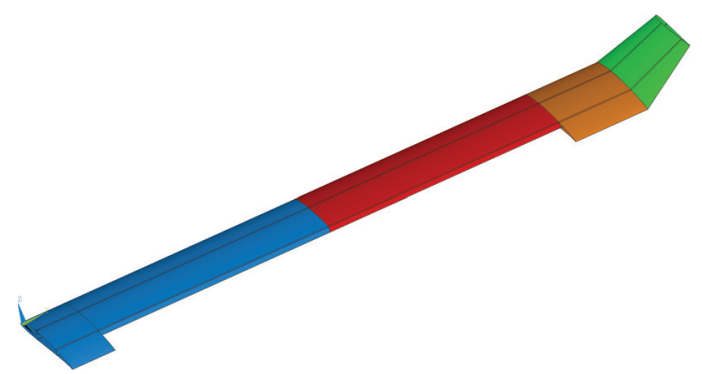

Figure 7: Fixed wing model with coloured sections, identifying the different skin laminate optimization areas.

Table 2: Parameter values used to create the design of experiments (baseline wing values in bold).

\begin{tabular}{|c|c|}
\hline$b, \mathrm{~m}$ & {$[2.665 \quad \mathbf{3 . 5 5 4}$} \\
\hline$c_{I F W}, \mathrm{~m}$ & $0.3210 .386]$ \\
\hline $\bar{l}_{\text {var }}$ & 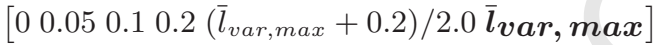 \\
\hline $\bar{c}_{f l a p}$ & {$\left[\begin{array}{ll}0.3 & 0.4\end{array}\right]$} \\
\hline$W, \mathrm{~N}$ & {$\left[\begin{array}{lll}120 & 150 & 180\end{array}\right]$} \\
\hline
\end{tabular}

In Table 2 the bold values correspond to the baseline wing dimensions and weight. As can be inferred from the same table, three values of span, IFW chord and aeroplane weight were selected. The values of span and weight were computed considering $\mathrm{a} \pm 25 \%$ variation centred in the baseline wing reference values. The minimum value of the IFW chord was set to the reference, in order to avoid unrealistic aspect ratios. The other values were considered to be 1.25 and 1.5 times higher than the baseline. In what concerns $\bar{l}_{\text {var }}$ parameter, a total of six values were selected: the zero value corresponds to the conventional wing configuration and the $\bar{l}_{v a r, \max }$ corresponds to the maximum VS ratio. The latter is a function of the span and the interface between the IFW and OMW $\left(l_{\text {over } 1}\right)$. Note that the baseline wing uses the larger VS ratio. Regarding $\bar{c}_{\text {flap }}$ parameter, only a higher value than the VSW reference value was added, in order to reduce the computational time. The parameters in study (independent variables) were used to create the wings to be optimized in ANSYS ${ }^{\circledR}$, by using sequential repetitions of unique parameters combinations.

There are some geometrical and cross-section dimensions that were kept constant throughout the study. Table 3 summarizes these constant geometrical and crosssection dimensions. As described in section 3.5 some dimensions were scaled using the baseline wing dimensions. These reference values are presented in Table 4 .

In the next sections, the results of the parametric study are presented. Since the parametric study is composed of a large dataset (324 parameter combinations), only a case study is shown using the baseline wing. Later in this section, the polynomial 
Table 3: Parametric study constant geometrical and cross-section dimensions.

\begin{tabular}{cccc}
\hline \multicolumn{2}{c}{ Geometric } & \multicolumn{2}{c}{ Cross-section } \\
\hline$l_{\text {fus }}$ & $0.12 \mathrm{~m}$ & $t_{\text {foam }, I F W}$ & $2 \mathrm{~mm}$ \\
$l_{\text {over } 2}$ & $0.025 \mathrm{~m}$ & $t_{\text {foam, } w \text { eb }, I F W}$ & $3 \mathrm{~mm}$ \\
$\Gamma_{\text {tip }}$ & $57^{\circ}$ & $t_{\text {foam }, \text { OMW }}$ & $2 \mathrm{~mm}$ \\
& & $t_{\text {foam }, w e b, O M W}$ & $2 \mathrm{~mm}$ \\
\hline
\end{tabular}

Table 4: Baseline wing geometrical parameters used in scaling.

\begin{tabular}{cc}
\hline Parameter & Value \\
\hline$l_{\text {over } 1, b l w}$ & $0.125 \mathrm{~m}$ \\
$l_{\text {tip }, \text { blw }}$ & $0.157 \mathrm{~m}$ \\
$c_{O M W, b l w}$ & $0.234 \mathrm{~m}$ \\
$c_{\text {tip }, \text { blw }}$ & $0.18 \mathrm{~m}$ \\
\hline
\end{tabular}

approximations for each studied case are analysed and the interaction between the parameters asserted.

\subsection{Design Loading Analysis}

According to what was introduced, a single reference wing was studied and loading values for different wings were generalized based on CS-VLA regulation gust loading.

Design loads were estimated with the use of the $\mathrm{V}$-n diagram as specified in EASA's CS-VLA [19]. This diagram allows to obtain the symmetrical load factor envelope for any given wing configuration as a function of speed. It was assumed that the maximum and minimum manoeuvre load factors in any wing configuration are +3 and -1.5 , respectively. Each diagram is composed of a manoeuvre envelope and a gust envelope. The gust speeds for the cruise, $V_{g, C}$, and dive, $V_{g, D}$, conditions are $15.24 \mathrm{~m} / \mathrm{s}$ and $7.62 \mathrm{~m} / \mathrm{s}$, respectively. The cruise speed was computed using Eq.(6) and the wing lift coefficients curve slopes using Eq.(11). The aerofoil lift curve slope was estimated using XFOIL [22]. It was assumed that the minimum wing lift coefficient is half the maximum wing lift coefficient. The maximum lift coefficient, $C_{L, \max }$, was assumed to be the same for all wings due to the Reynolds number and aerofoil similarity among the three wing configurations. This coefficient, along with lift-to-drag ratio and moment coefficient were estimated based on an aerodynamic analysis using Vortex Lattice Method (VLM) performed in XFLR5. The data required to construct the V-n diagrams is summarized in Table 5.

A total of three V-n diagrams were calculated, one for each wing configuration. Figure 8 shows the V-n diagram for each wing configuration superimposed. Referring 
Table 5: Required data to compute the V-n diagrams of the three wing configurations.

\begin{tabular}{cccccc}
\hline $\begin{array}{c}\text { Wing } \\
\text { span }\end{array}$ & $\begin{array}{c}\text { Wing } \\
\text { area, m }\end{array}$ & $C_{L, \max }$ & $C_{L, \text { min }}$ & $\begin{array}{c}C_{L_{\alpha}}, \\
\mathrm{rad}^{-1}\end{array}$ & $\begin{array}{c}\text { Design cruise } \\
\text { speed, m/s }\end{array}$ \\
\hline Max. & 0.913 & 1.44 & -0.72 & 5.062 & 30.76 \\
Int. & 0.798 & 1.44 & -0.72 & 4.978 & 32.91 \\
Min. & 0.682 & 1.44 & -0.72 & 4.870 & 35.59 \\
\hline
\end{tabular}

to Fig.8, the critical case for the maximum, intermediate and minimum span wings is highlighted using a circle, a delta and a square symbol, respectively. Because the negative load factors are much lower than the positive ones those are not considered. The critical envelope in every case is, as expected, the gust envelope. Observing the three diagrams, the critical envelope is that of the fully extended wing, since it presents the higher load factor. The referred point corresponds to the speed and load factor computed with Eqs.(13) and (14), respectively. Therefore, in the parametric study maximum span configurations were used in all studied cases.

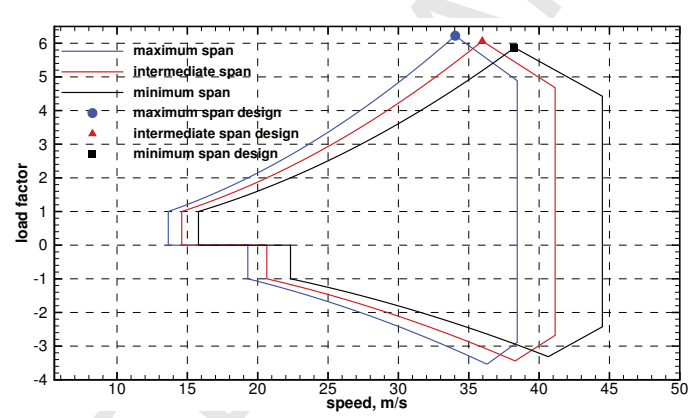

Figure 8: V-n diagrams for each wing configuration.

The presented analysis was generalized for all wing configurations and the lift, drag and pitching moment were calculated for the given speed, wing area and aeroplane weight, using Eqs.(15), (16) and (17), respectively. Recalling Eq.(16), the drag is computed by scaling the lift-to-drag ratio of the baseline, $(L / D)_{b l w}$. A value of 32.6 was used. Regarding the pitching moment, $C_{M}$, a constant value of -0.15 was used for all the studied wings. The aerodynamic reference values are summarized in Table 6 .

Table 6: Baseline wing aerodynamic scaling parameters.

\begin{tabular}{cc}
\hline Parameter & Value \\
\hline$(L / D)_{b l w}$ & 32.6 \\
$C_{M, b l w}$ & -0.15 \\
\hline
\end{tabular}

Since the effect of AOA was neglected, lift and drag are already the vertical and 
horizontal forces, respectively. The vertical force and pitching moment were moved to the spar web fore and aft positions using Eqs.(18) and (19), respectively. Finally, the horizontal force was divided by the four spar cap corners.

\subsection{Baseline Wing Mesh Convergence Study}

A convergence analysis of the finite element model of the baseline wing was carried out to assess the sensitivity of the maximum tip displacement and rotation as functions of the element number in the mesh. Several meshes were created and a static analysis was performed with the loading distributed along the span. It should be highlighted that, due to the nature of the parametric study, wing geometry changes depending on input parameters. In order to avoid the time consuming process of performing a mesh study for each geometry configuration, the relative spacing between elements was kept constant across all FEM analysis.

\subsection{Baseline Wing Optimization and Analysis}

The focus of this section is to assess the functionality and correctness of the FEM and optimization scripts developed in ANSYS ${ }^{\circledR}$ APDL. To achieve this, the baseline wing underwent structural optimization and subsequent structural analysis of the optimized structure.

Since a prototype of the baseline wing is planned to be built and installed in a prototype RPAS, one should take in consideration the minimum bounds of the design variables. Regarding the skin laminate, the minimum acceptable value is $0.12 \mathrm{~mm}$, corresponding to a layer of $185 \mathrm{~g} / \mathrm{m}^{2}$ plain weave carbon/epoxy. Thus, both $t_{l a m, I F W}$ and $t_{l a m, O M W}$ minimum optimization bounds were set to this value. The spar widths minimum bounds were selected based on standard available sections from vDijk high-strength pultrusions [23]. In that list, the minimum available section is $2 \mathrm{~mm} \times 0.4 \mathrm{~mm}$, being this value used as lower limit to $w_{s c, I F W}$ and $w_{s c, O M W}$.

Using the above considerations, the optimization was run with the baseline wing specifications. In Table 7 one can see the initial and final values of the design variables and of the objective and constraint functions; and in Fig.9 the variation of the same variables throughout the design sets. The initial values of the design variables were selected so that the wing had sufficient stiffness to guarantee a feasible solution, i.e., all constrains were fulfilled. 
Table 7: Baseline wing design variables, and objective and constraint functions initial and final values.

\begin{tabular}{ccccccccc}
\hline & $\begin{array}{c}w_{s c, I F W}, \\
\mathrm{~mm}\end{array}$ & $\begin{array}{c}t_{\text {lam }, I F W}, \\
\mathrm{~mm}\end{array}$ & $\begin{array}{c}w_{\text {sc,OMW }}, \\
\mathrm{mm}\end{array}$ & $\begin{array}{c}t_{\text {lam, }, O M W}, \\
\mathrm{~mm}\end{array}$ & $\begin{array}{c}m_{\text {wing }}, \\
\mathrm{kg}\end{array}$ & $\begin{array}{c}w_{\text {tip }}, \\
\mathrm{m}\end{array}$ & $\begin{array}{c}\theta_{\text {tip }}, \\
\mathrm{deg}\end{array}$ & $\begin{array}{c}\overline{S R}_{T W}, \\
\%\end{array}$ \\
\hline Initial & 50.0 & 0.48 & 40.0 & 0.13 & 2.54 & 0.028 & -0.09 & 0.0073 \\
Final & 21.7 & 0.12 & 0.8 & 0.12 & 1.00 & 0.088 & -0.36 & 0.0113 \\
\hline
\end{tabular}

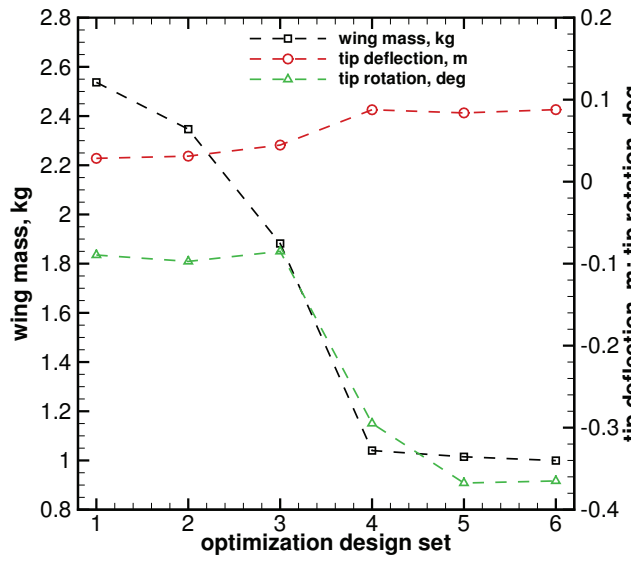

(a)

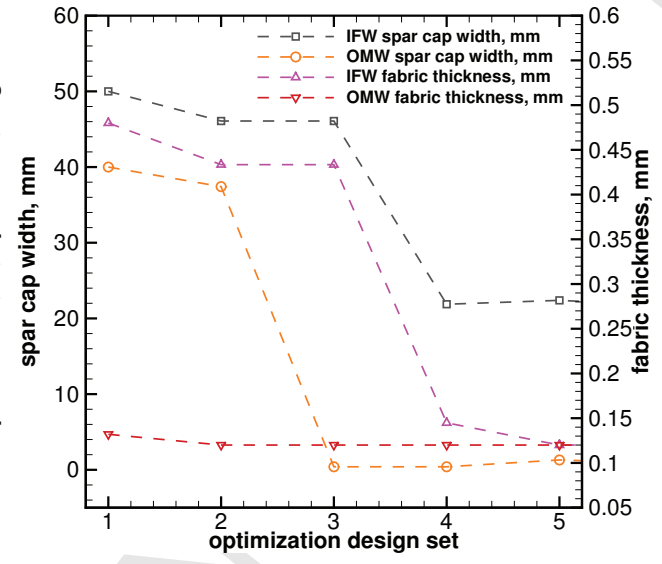

(b)

Figure 9: Baseline wing optimization design: (a) objective/constraint variables and (b) design variables.

Observing Fig.9, one can see that the wing mass was significantly reduced from $2.54 \mathrm{~kg}$ to $1 \mathrm{~kg}$, indicating that the optimization started with design variables that were relatively far from the optimum. This can be confirmed from the constraint variables, $w_{t i p}$ and $\theta_{t i p}$, that are $0.028 \mathrm{~m}$ and $-0.09^{\circ}$ in the first set and $0.088 \mathrm{~m}$ and $-0.36^{\circ}$ in the final set. The upper bound of the tip deflection $(0.088 \mathrm{~m}$ or $0.025 b)$ was reached, whereas the lower bound of the tip rotation $-0.6^{\circ}$ was not achieved. The latter happened since the skin laminates resist the majority of the torsion loading and they were limited by manufacturing requirements. This indicates that a thinner laminate could further lower the mass. On the other hand, the spar caps resist the majority of the bending loading and due to their higher design space, the tip deflection constraint was reached. Regarding the wing strength, it was not problematic since the $\overline{S R}_{T W}$ is not near the constraint value of $0.1 \%$, being nearly ten times lower.

Using the optimized fabric thicknesses and spar cap widths, the wing was analyzed in FEM. Wing surface plots of the vertical displacements and failure criterion were obtained to perform a detailed analysis. The former can be seen in Fig.10. The referred figure shows a displacement distribution which smoothly increases from root (on the left hand side) to tip. The displacement reaches a maximum of $0.088 \mathrm{~m}$ at the 
wing tip, being around $2.5 \%$ of the span. As expected, the maximum twist appears at the wing tip. Due to the relative magnitude of the fore web and aft web vertical loads the VSW demonstrates a positive twist.

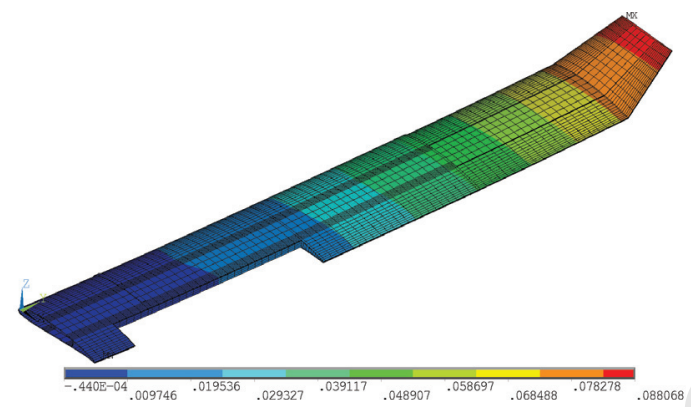

Figure 10: Vertical displacements of the optimized baseline VSW (displacements in m).

Figure 11 shows the inverse of $T s a i-W u$ strength ratio criterion of the baseline VSW, for the various material layers: outer sandwich laminate, sandwich core (foam and pultruded carbon/epoxy) and inner sandwich laminate.

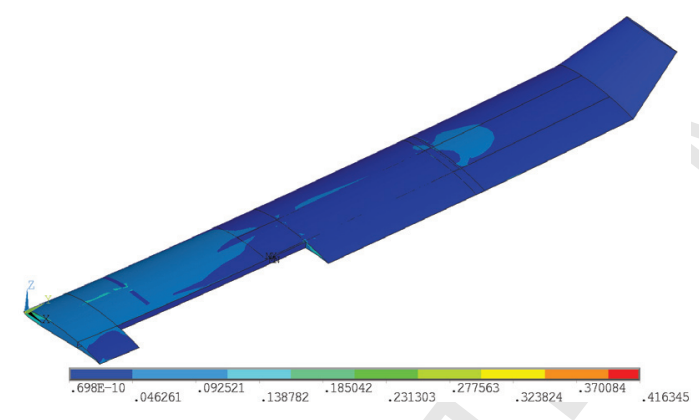

(a)

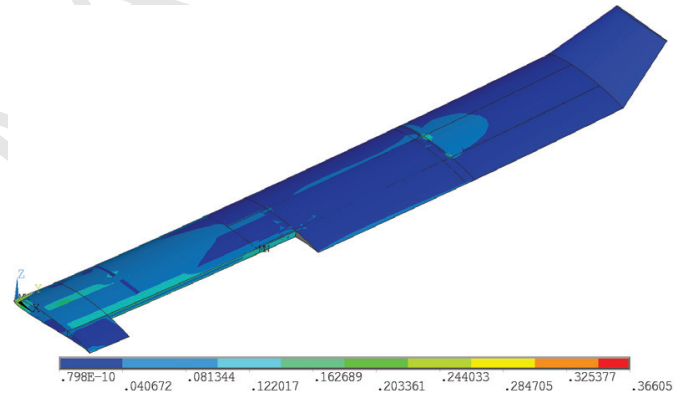

(b)

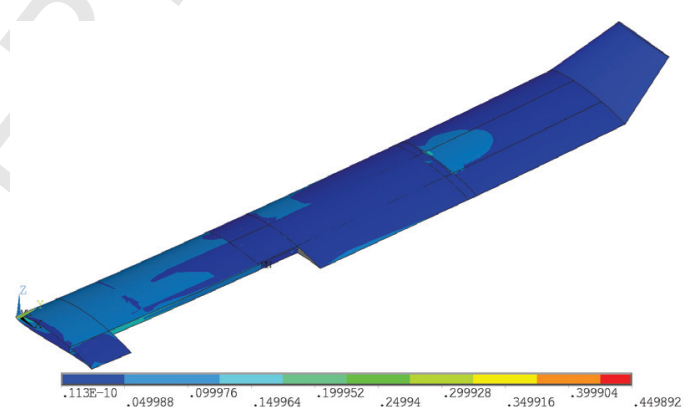

(c)

Figure 11: Inverse of Tsai-Wu strength ratio of the optimized baseline VSW: (a) outer sandwich laminate, (b) sandwich core (foam and pultruded carbon/epoxy) and (c) inner sandwich laminate.

Observing Fig.11, it is possible to conclude that both IFW and OMW are oversized since the failure criterion never exceeds unity, being about 0.45 near the wing root. The more stressed areas of the IFW are located near the root and in the region of $l_{\text {over } 1}$ contact zone. In particular, the maximum failure criterion near the root area 
was to be expected since it is there that the maximum bending moment is present, along with the flap discontinuity, that creates a stress concentration. Regarding the moving portion of the VSW, one can see that it is relatively oversized, as evidenced by the even lower failure criterion. The more stressed areas are seen in the end of the second contact region $\left(l_{\text {over } 2}\right)$ between the two wing elements, being more pronounced in the location of the spar caps.

In conclusion, the generalized lightly loaded structure results from the fact that the minimum thicknesses allowed in the skin composite laminate were $0.12 \mathrm{~mm}$, specially in the OMW, where the loads are much lower. It should be added that the use of bonded contact formulation reduced the accuracy of stress predictions near the contact zones. In fact, in the $l_{\text {over } 1}$ and $l_{\text {over } 2}$ areas the surfaces are bonded and no stress concentration can be identified due to the normal pressure effects. In previous works $[24,25]$, the use of a standard contact formulation clearly identified those zones with stress concentrations near the contact region.

\subsection{Parameter Influence and Mass Estimation}

The nonlinear ERR-Causality method was used to derive three multivariable second order polynomials: reference fixed-wing, VSW and VSW to fixed wing ratio.

The used approximation method includes an arrangement of the significant terms from higher to lower significance. It is expected that using higher number of terms, the polynomial fitting precision would increase. However, a synthetic and accurate polynomial equation is desired, since it simplifies its use. To simplify the polynomials a study of the SERR was performed. The variation of SERR was computed with increasing number of terms, until the variation was lower than a convergence stopping criterion of $0.1 \%$. A SERR value equal to $100 \%$ would stand for an ideal regression where there is a perfect relationship between the computed and the predicted data.

After computation of the polynomials, the influence of each parameter can be determined by a careful analysis of the obtained equations. Note that, the independent variables of the polynomials were considered as the difference between a given parameter and its average over the design space. Three-dimensional plots were produced to perform a graphical exploration of the most significant parameter's interactions. 
In the next sections, the analysis of each polynomial approximation is performed.

\subsubsection{Fixed Wing}

Table 8 shows the SERR study of the fixed wing polynomial. One can infer that SERR increases with increasing number of terms and that its variation is not linear. This is due to the different significance of each term. The convergence occurs for 12 terms, since $\triangle$ SERR decreases below the convergence stopping criterion of $0.1 \%$ $(0.04 \%)$. The increment in precision in the next set is $0.02 \%$, being also below the convergence stopping criterion. It is important to analyse at least a further term, since the variation of SERR can occur in a lightly dampened oscillatory behaviour. The resulting SERR gives $99.72 \%$ of the solution, being close to $100 \%$, which indicates that the strength of association between the variables is high and the method is adequate to fit the data. Note that with the complete polynomial, the SERR is $99.74 \%$, thus, very little $(<0.2 \%)$ would be gained by adding more terms.

Table 8: Fixed wing polynomial SERR calculated for different number of terms (convergence shown in bold).

\begin{tabular}{cccccc}
\hline & 9 & 10 & 11 & $\mathbf{1 2}$ & 13 \\
\hline SERR & $99.01 \%$ & $99.52 \%$ & $99.68 \%$ & $\mathbf{9 9 . 7 2 \%}$ & $99.74 \%$ \\
$\Delta$ SERR & $0.26 \%$ & $0.51 \%$ & $0.16 \%$ & $\mathbf{0 . 0 4 \%}$ & $0.02 \%$ \\
\hline
\end{tabular}

Error metrics of the polynomial fitting were assessed and it was concluded that the second order polynomial produces an adequate fit of the data. The maximum relative error was below $10 \%$ and the maximum absolute error below $0.050 \mathrm{~kg}$. The latter is a low value, considering that the minimum computed wing mass is $0.259 \mathrm{~kg}$.

The resultant polynomial can be seen in Eq.(31). The terms are organized in decreasing order of significance, facilitating the identification of the most significant ones.

$$
\begin{aligned}
m_{s t r, f w}= & f\left(b, c_{I F W}, \bar{c}_{\text {flap }}, W\right) \\
= & 0.3546(b-3.554)-1.5466\left(c_{I F W}-0.321\right)+0.002768(W-150) \\
& -1.2893(b-3.554)\left(c_{I F W}-0.321\right)-0.8358\left(\bar{c}_{\text {flap }}-0.35\right) \\
& +0.002076(b-3.554)(W-150)-0.01322\left(c_{I F W}-0.321\right)(W-150) \\
& -0.4256(b-3.554)\left(\bar{c}_{\text {flap }}+0.06819(b-3.554)^{2}-22.007\left(\bar{c}_{\text {flap }}-0.35\right)^{2}\right. \\
& +5.9577\left(c_{I F W}-0.321\right)^{2}-0.00469(W-150)\left(\bar{c}_{\text {flap }}-0.35\right)+0.6186
\end{aligned}
$$

In Eq.(31), the parameter $(b-3.554)$ in the first term represents the difference between the given span and the average of the spans given in Table 2 . The other terms 
follow a similar reasoning. Analysing the referred equation, one may acknowledge that span and chord (first and second terms) have the higher significance, accounting for $86.16 \%$ of the total significance. The high influence of $b$ and $c_{I F W}$ in the wing mass was already expected. In fact, a higher aspect ratio (higher span and/or lower chord) have the effect of increasing the root bending moment, demanding extra structural mass to comply with the structural constraints. The aeroplane weight is also important since wing loading is increased and, consequently, root bending moment increases. The additional linear parameter, flap chord ratio appears to be inversely correlated with wing mass. Therefore, an increase in flap chord, reduces the wing mass. However, caution should be taken, since the actual flap mass was not included in the study.

In order to visually analyse the interaction of parameters, three-dimensional plots were produced. Only the two most significant interactions are presented: span $\times$ IFW chord and span $\times$ weight. The data computed using the parametric study and the data approximated by the nonlinear regression are overlapped and illustrated in the form of scatter and surface plots, respectively. Figure 12 illustrates the threedimensional plots of span and chord for the two weights, with constant flap chord ratio, and Fig.13 the plots of span and weight for the two studied chords, with constant flap chord ratio.

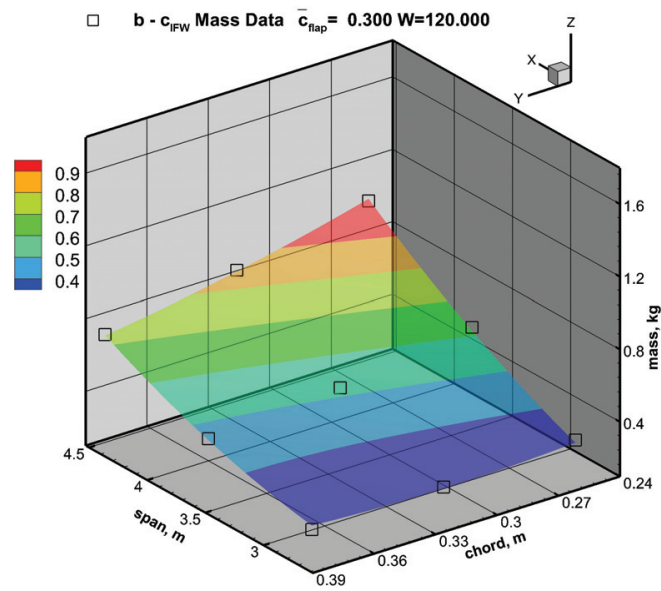

(a)

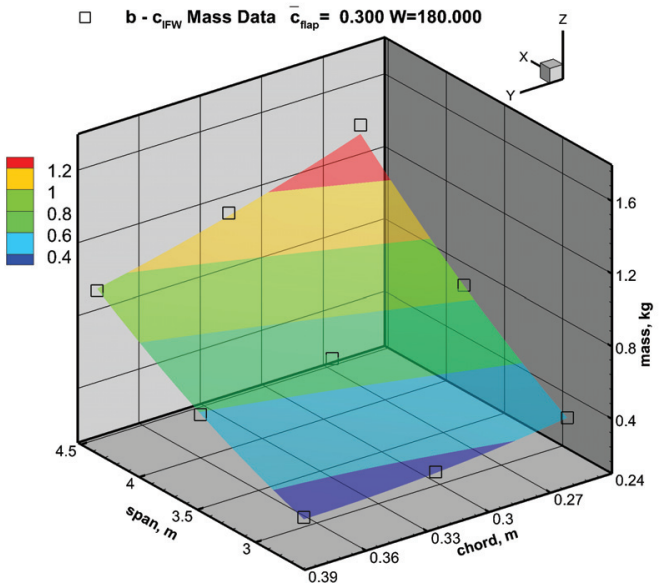

(b)

Figure 12: Fixed wing mass predictions and actual data points as functions of span and chord for the two studied weighs $\left(\bar{c}_{\text {flap }}=0.3\right)$ : (a) $120 \mathrm{~N}$ and (b) $180 \mathrm{~N}$.

Looking at Figs.12(a) to (b), one can verify an increase in mass with increasing 
span and decreasing chord. Increasing span, augments root bending moment, while lowering the chord reduces the section inertia, requiring more structural material, to achieve the same stiffness and strength. The trends are similar for the two aeroplanes weights, but the magnitudes vary considerably, as expected. In fact, for the lower aeroplane weight, the maximum wing mass is $1.03 \mathrm{~kg}$, whereas for the higher weight, the maximum mass is $1.37 \mathrm{~kg}$. However, the minimum wing mass is approximately similar for the two MTOWs, occurring for minimum span and maximum chord. The minimum mass varies between $0.332 \mathrm{~kg}$ and $0.376 \mathrm{~kg}$.

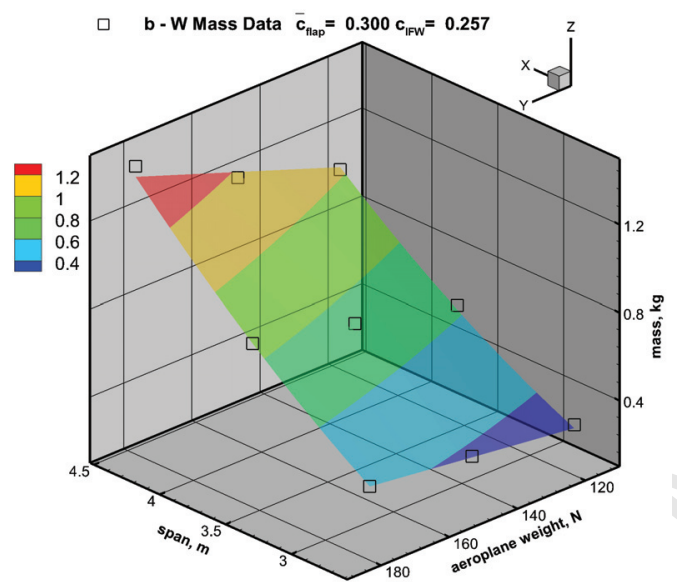

(a)

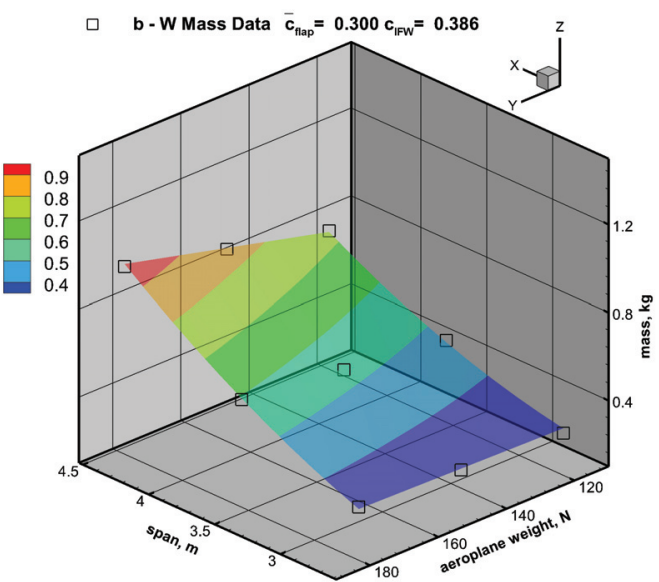

(b)

Figure 13: Fixed wing mass predictions and actual data points as functions of span and weight for the two studied wing chords $\left(\bar{c}_{\text {flap }}=0.3\right)$ : (a) $0.257 \mathrm{~m}$ and (b) $0.386 \mathrm{~m}$.

Observing Figs.13(a) to (b), one can see the same trend for all figures: increasing span and MTOW increases wing mass. The span increase has more impact in the wing mass than the MTOW variation, as indicated by the higher slope of the former. Maximum mass occurs for the smaller chord (Fig.13(a)), being $1.37 \mathrm{~kg}$. As in the previous analysed figures, the minimum mass is approximately similar irrespective of the chord, varying between $0.335 \mathrm{~kg}$ and $0.363 \mathrm{~kg}$, but occurs for minimum MTOW and span. One should also add that the variation is nearly linear, as evidenced by the near planar surfaces.

\subsubsection{Variable-span Wing}

Table 9 shows the SERR study of the VSW polynomial. Analysing the table, one can see that the convergence occurs for 13 terms, since $\triangle$ SERR decreases below the convergence stopping criterion of $0.1 \%(0.09 \%)$. The increment in precision in the 
next set is $0.06 \%$, being also below the convergence stopping criterion. The resulting SERR with 13 terms gives $99.41 \%$ of the solution (total is $99.58 \%$ ). As in the fixed wing polynomial, the resulting SERR value is close to $100 \%$, which indicates that the strength of association between the variables is high and the method is adequate to fit the data.

Table 9: VSW polynomial SERR calculated for different number of terms (convergence shown in bold).

\begin{tabular}{cccccc}
\hline & 10 & 11 & 12 & $\mathbf{1 3}$ & 14 \\
\hline SERR & $98.84 \%$ & $99.11 \%$ & $99.33 \%$ & $\mathbf{9 9 . 4 1 \%}$ & $99.47 \%$ \\
$\Delta$ SERR & $0.37 \%$ & $0.27 \%$ & $0.23 \%$ & $\mathbf{0 . 0 8 \%}$ & $0.06 \%$ \\
\hline
\end{tabular}

Additional error metrics of the polynomial fitting were assessed. The maximum relative error is below $12 \%$ and the maximum absolute error is about $0.14 \mathrm{~kg}$, which is a relatively high value. However, the RMSE error is only $0.023 \mathrm{~kg}$, which indicates that the majority of the data points have a significantly lower value.

The resultant polynomial is presented in Eq.(32). As in the previous polynomial, the terms are organized in decreasing order of significance.

$$
\begin{aligned}
m_{\text {str }, V S W}= & f\left(b, c_{I F W}, \bar{l}_{\text {var }}, \bar{c}_{\text {flap }}, W\right) \\
= & 0.3632(b-3.554)-1.2557\left(c_{I F W}-0.321\right)+0.002496(W-150) \\
& -1.4998(b-3.554)\left(c_{I F W}-0.321\right)-0.8066\left(\bar{c}_{\text {flap }}-0.35\right) \\
& +0.001875(b-3.554)(W-150)-0.4752(b-3.554)\left(\bar{c}_{\text {flap }}-0.35\right) \\
& +0.07991(b-3.554)^{2}-28.002\left(\bar{c}_{\text {flap }}-0.35\right)^{2} \\
& +9.1823\left(c_{I F W}-0.321\right)^{2}-0.01185\left(c_{I F W}-0.321\right)(W-150) \\
& +3.5632\left(c_{I F W}-0.321\right)\left(\bar{l}_{\text {var }}-0.153\right)-1.0233\left(\bar{l}_{\text {var }}-0.153\right)+0.6893
\end{aligned}
$$

Analysing Eq.(32) it is possible to conclude that the presented polynomial is similar to the fixed wing polynomial (Eq.(31)). The high influence of wingspan, weight and IFW chord on the wing mass is verified, both in linear and non-linear contributions. Interestingly, the VS ratio has a small contribution to the overall solution $(0.3 \%)$. This is somewhat intriguing and is probably explained by the larger contribution of the other parameters, which effectively mask the effect of span change. This also indicates that the morphing interface is more complicated than anticipated and that the structural duplication, resulting from the IFW/OMW interface, could not be as significant as expected. Thus, further considerations about the influence of span change have to be deferred to the VSW to fixed wing ratio polynomial. The 
flap chord ratio is again inversely correlated with wing mass. However, as in the fixed wing case, caution should be taken, since the actual flap mass was not included in this study.

Similarly to the previous studied case, three-dimensional plots were produced. The most significant interaction is presented, being span $\times$ IFW chord. To aid the comprehension of the impact of the VS ratio, span is plotted against the latter. The data computed in the parametric study and the data approximated using the nonlinear regression are overlapped and illustrated in the form of scatter and surface plots, respectively. Figure 14 illustrates the plots of span and chord for two extreme VS ratios, with constant flap chord ratio and weight, and Fig.15 the three-dimensional plots of span and VS ratio for two IFW chords, with the remaining parameters constant.

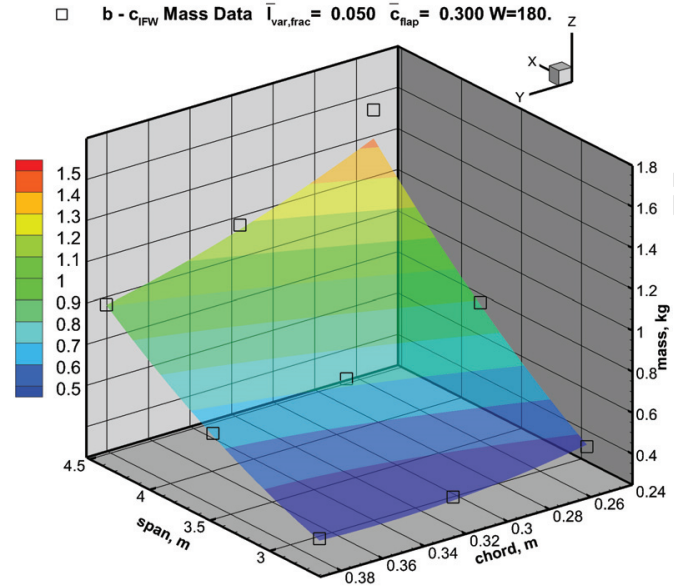

(a)

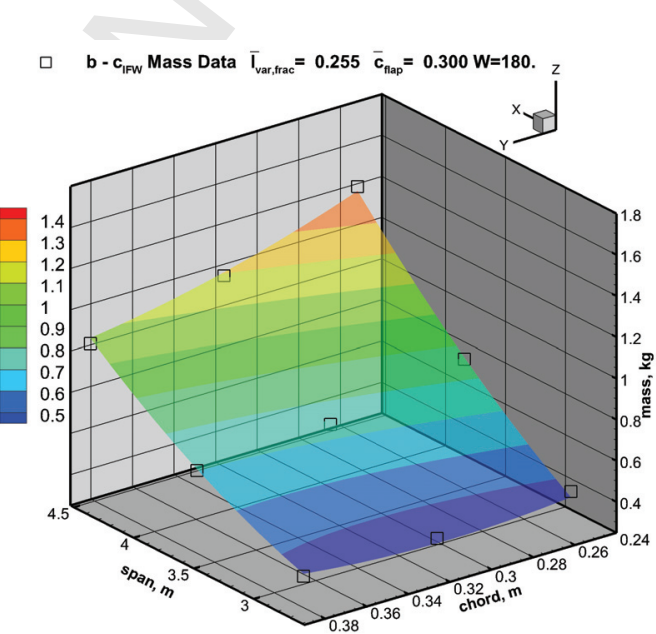

(b)

Figure 14: VSW mass predictions and actual data points as functions of span and IFW chord for two VS ratios $\left(\bar{c}_{\text {flap }}=0.3\right.$ and $W=180 \mathrm{~N}$ ): (a) 0.05 and (b) 0.255 .

Observing Figs.14(a) and (b), it is possible to see that the increase of the VS ratio causes a small decrease in the wing mass. The two figures show the same trends: the increase in span and the decrease in chord increases the wing mass. However, the span effect is slightly more pronounced than the chord effect. Additionally, the chord effect is more pronounced for higher span values. Therefore, higher mass occurs for maximum span and minimum wing chord. The maximum wing mass is $1.429 \mathrm{~kg}$ and $1.407 \mathrm{~kg}$ for the lower and higher VS ratios, respectively.

Figures $15(\mathrm{a})$ to (b) show that the increase in chord causes a decrease in wing 


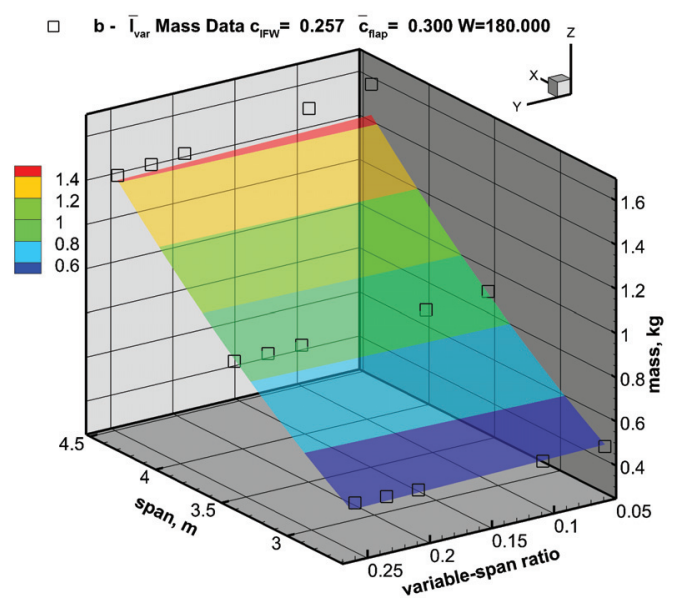

(a)

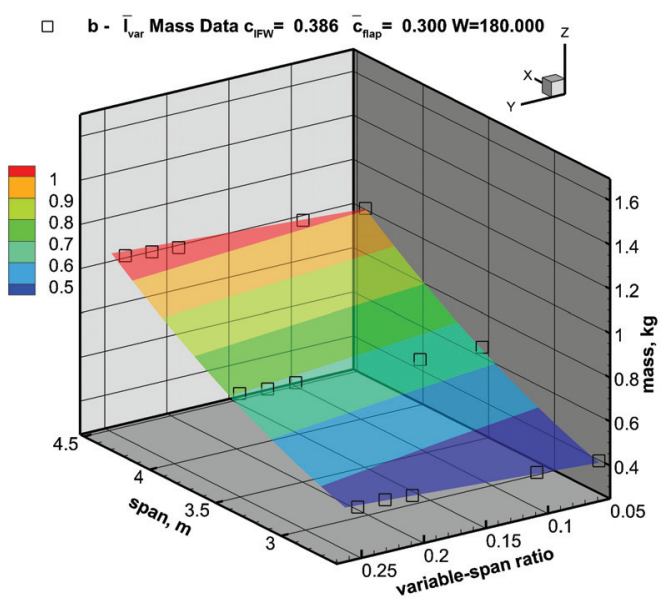

(b)

Figure 15: VSW mass predictions and actual data points as functions of span and VS ratio for two studied IFW chords $\left(\bar{c}_{\text {flap }}=0.3\right.$ and $W=180 \mathrm{~N}$ ): (a) $0.257 \mathrm{~m}$ and (b) $0.386 \mathrm{~m}$.

mass. The span has the highest influence on the wing mass, with the mass increasing with span. As anticipated from the polynomial analysis, the influence of the VS ratio is almost negligible, as evidenced by the near horizontal surfaces, in the VS ratio axis. Despite this, the lower chord shows a small mass reduction with increasing VS ratio, whereas the higher chord shows a mass increase. Therefore, the trend inverts between these two chords. This could be explained by the available area to transfer the loading between the IFW/OMW: the lower chord has less area and the larger chord has more area. It should be added that, the higher wing mass occurs for the maximum span, minimum VS ratio and minimum chord. The maximum wing mass reaches $1.429 \mathrm{~kg}$ for the smaller chord and $1.005 \mathrm{~kg}$ for the larger chord.

\subsubsection{Variable-span Wing Mass Ratio}

Similar to the previous polynomials, a SERR study was performed. Table 10 shows the SERR study of the VSW to fixed wing polynomial. Analysing the table, one can see that convergence occurs for 16 terms with a $\triangle \mathrm{SERR}$ of $0.07 \%$. The resulting SERR with 16 terms corresponds to $97.41 \%$ of the solution, being the maximum SERR $97.46 \%$. Contrary to the fixed wing and VSW polynomials, the resulting SERR value is not as close to $100 \%$, indicating a slightly weaker association between variables.

Additional error metrics of the polynomial fitting were assessed, being evident that the approximation has sufficient accuracy. The maximum relative error is below 
Table 10: VSW to fixed wing ratio polynomial SERR variation for different number of terms (convergence shown in bold).

\begin{tabular}{cccccc}
\hline & 13 & 14 & 15 & $\mathbf{1 6}$ & 17 \\
\hline SERR & $96.36 \%$ & $96.88 \%$ & $97.34 \%$ & $\mathbf{9 7 . 4 1 \%}$ & $97.44 \%$ \\
$\Delta$ SERR & $0.61 \%$ & $0.54 \%$ & $0.48 \%$ & $\mathbf{0 . 0 7 \%}$ & $0.03 \%$ \\
\hline
\end{tabular}

$5 \%$ and RMSE error is 0.016 , which indicates that the majority of the data points have significantly lower error values.

The resultant polynomial is presented in Eq.(33), being the terms organized in decreasing order of significance.

$$
\begin{aligned}
& m_{\text {str }, V S W / f w}=f\left(b, c_{I F W}, \bar{l}_{\text {var }}, \bar{c}_{\text {flap }}, W\right)=-0.06964(b-3.554) \\
& \quad+0.9585\left(c_{I F W}-0.321\right)-0.6468(b-3.554)\left(c_{I F W}-0.321\right) \\
& \quad+0.3507\left(\bar{l}_{\text {var }}-0.153\right)+6.1716\left(c_{I F W}-0.321\right)\left(\bar{l}_{\text {var }}-0.153\right) \\
& \quad-0.0009336(W-150)-0.3275(b-3.554)\left(\bar{l}_{\text {var }}-0.153\right) \\
& \quad+0.2809\left(\bar{c}_{\text {flap }}-0.35\right)+0.0400(b-3.554)^{2}+3.8598\left(c_{I F W}-0.321\right)^{2} \\
& \quad+1.8603\left(\bar{l}_{\text {var }}-0.153\right) \bar{c}_{\text {flap }}+2.6966\left(c_{I F W}-0.321\right)\left(\bar{c}_{\text {flap }}-0.35\right) \\
& \quad-0.1838(b-3.554)\left(\bar{c}_{\text {flap }}-0.35\right) \\
& \quad-0.003389\left(\bar{l}_{\text {var }}-0.153\right)(W-150)+0.7316\left(\bar{l}_{\text {var }}-0.153\right)^{2}+1.1066
\end{aligned}
$$

Equation (33) show that, again, span and chord have the higher effect, both with linear and non-linear contributions. However, contrary to the previous polynomial, the VS ratio has now a significant contribution appearing has a linear parameter and also combined with chord. This corroborates the fact that the other parameters were effectively masking the effect of the span variation ratio. The impact of the latter is complex, being the three-dimensional plots crucial to interpret the influence between parameters. Two interactions are presented: span $\times$ IFW chord and span $\times$ VS ratio. Figure 16 illustrates the three-dimensional plots of span and IFW chord for two VS ratios, with constant flap chord ratio and aeroplane weight, and Fig.17 the three-dimensional plots of span and VS ratio for two IFW chords, with constant flap chord ratio and aeroplane weight.

Figure 16(a) to (b) demonstrates an interesting and complex influence of span and chord. Different trends are visible for the two VS ratios. Among the four studied VS ratios (two intermediate cases not shown), the mass prediction surface appears to rotate along a diagonal line that crosses the minimum span and maximum chord points, in the sense of increasing the maximum mass penalty, while keeping the minimum approximately constant. Thus, higher mass penalties occur for minimum 


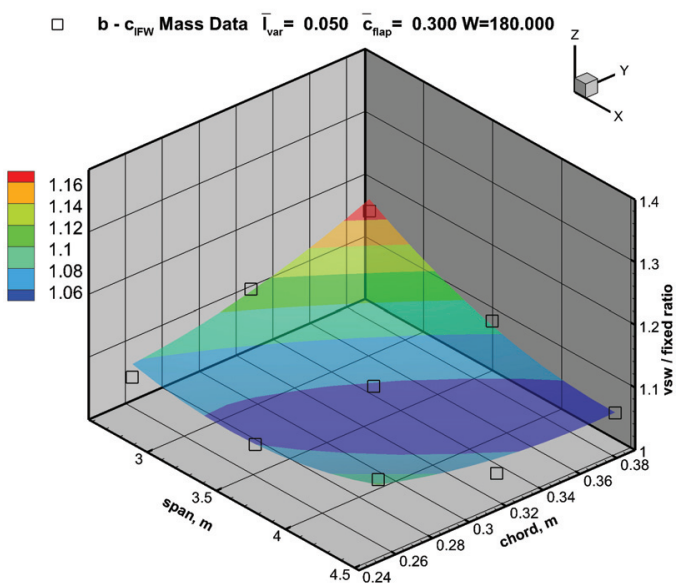

(a)

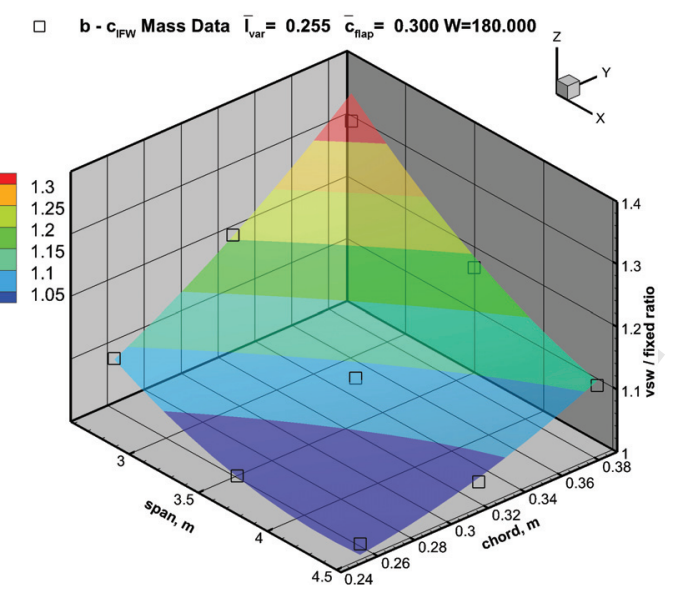

(b)

Figure 16: VSW to fixed wing ratio mass predictions and actual data points as functions of span and IFW chord for two VS ratios $\left(\bar{c}_{\text {flap }}=0.3\right.$ and $\left.W=180 \mathrm{~N}\right):(\mathrm{a}) 0.05$ and (b) 0.255 .

span and maximum chord. On the other hand, for each span ratio appears to exist a combination of span and chord that minimizes the mass penalty of the morphing wing. For $\bar{l}_{v a r}=0.05$, the minimum occurs for an intermediate chord and span, whereas for $\bar{l}_{v a r}=0.255$, maximum span and minimum chord grants the minimum mass penalty. The maximum penalty reaches 1.177 for the lower VS ratio and 1.350 for the higher VS ratio.

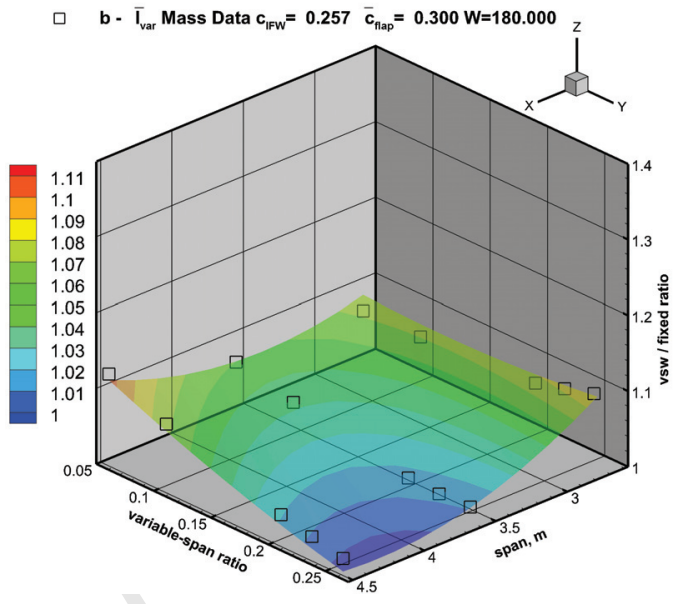

(a)

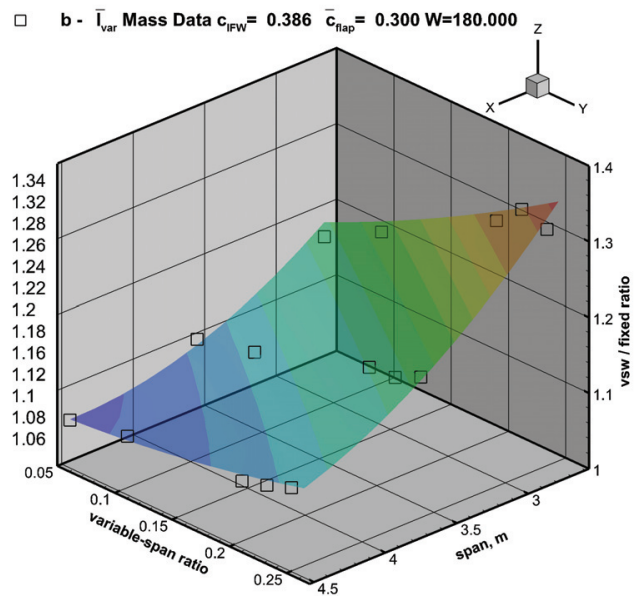

(b)

Figure 17: VSW to fixed wing ratio mass predictions and actual data points as functions of span and VS ratio for two IFW chords $\left(\bar{c}_{\text {flap }}=0.3\right.$ and $W=180 \mathrm{~N}$ ): (a) $0.257 \mathrm{~m}$ and (b) $0.386 \mathrm{~m}$.

Observing Fig.17(a) till (b), one can see distinct trends for the two chords. The lower chord has two peaks of higher mass penalty for minimum span and maximum VS ratio and vice-versa. In the second chord, only one peak exists and is more 
marked, occurring for minimum span and maximum $\bar{l}_{v a r}$, being the mass penalty 1.350. In fact, a rotation of the surfaces about the maximum span, minimum VS ratio diagonal is observed. Therefore, it is not a straightforward task to conclude which VS ratio minimizes the mass penalty for a given span and chord. However, the plots show that for a given span and chord, there is a $\bar{l}_{\text {var }}$ that minimizes the mass penalty of using the VSW. The maximum penalty reaches 1.104 for the smaller chord.

\section{Concluding Remarks}

In this paper, the influence of geometrical and inertial parameters on the structural mass of a VSW concept with an integrated trailing edge device was studied. Mass and mass ratio functions were created by fitting multivariable polynomials: fixed wing mass, VSW mass and VSW to fixed wing mass ratio. The polynomial approximations were derived based on a parametric study, which analysed the impact of wingspan, wing chord, span variation ratio, flap chord ratio and aeroplane weight. Then, a minimum mass optimization problem was developed in ANSYS ${ }^{\circledR}$ with stiffness and strength constraints, being the design variables structural thicknesses and widths. A parametric structural FEM was built to allow the structural analyses to be performed. The study was done for a conventional fixed wing and the VSW, which were then combined to ascertain the VSW mass increment, i.e., the mass penalization of the adopted morphing concept. The polynomials were found to produce good approximations of the wing mass. Later, the effects of the various VSW design parameters in the structural mass were inferred and synthesized. On one hand, it was found that span and chord have the highest impact in the wing mass. On the other hand, the VSW to fixed wing mass ratio proved that the influence of VS ratio in the wing mass is more complex than anticipated. In fact, it was found that the mass increase does not grow proportionally with span variation ratio and that, for each combination of span and chord, there exists a span variation ratio that minimizes the mass penalty.

The work presented showed that the conceptual idea behind the estimation of the morphing wing mass is relatively straightforward. However, implementation of the overall process revealed some problematic areas. Probably the most problematic area 
was the sizing loads. The current work used the CS-VLA specification to derive credible loads. However, these are only applicable for low wing loading aeroplanes and in the speed range of the CS-VLA regulation (Mach numbers below 0.3). In the present formulation the loads for a given aircraft mass are only affected via the analysis of the $\mathrm{V}$-n diagram and an assumption for L/D. Therefore, it is possible to expand the current method to higher Mach numbers by simply changing to appropriate V-n diagrams for a given design.

\section{Future Work}

In the near future, the newly developed polynomial functions can be used to derive a mass model, by adding the mass contributions of the span actuation system and morphing flap. Equations to estimate flap mass and span variation actuation system would have to be developed, using semi-empirical information and/or using FEM simulations. This mass estimation model could then be compared with an existing VSW design, namely the one in Reference [14]. However, a systematic comparison would have to be performed in order to prove its applicability over a wide range of VSWs designs.

\section{Acknowledgements}

The work presented herein was made possible by the financial support of the Portuguese Science Foundation (FCT - Fundação para a Ciência e Tecnologia) through the PhD Grant SFRH/BD/90159/2012.

The work presented herein has been partially funded by the European Community's Seventh Framework Programme (FP7) under the Grant Agreement 314139. The CHANGE project (Combined morphing assessment software using flight envelope data and mission based morphing prototype wing development) was a Level 1 project funded under the topic AAT.2012.1.1-2. involving 9 partners. The project started on August $1^{\text {st }}$ 2012, and ended in December 2015, lasting for a total of 40 months. 


\section{References}

[1] C. Hannon, L. Agyepong, V. Toropov, O. Querin, An Alternative View on Weight Estimation for the Aircraft Industry: Problems and MDO Solutions, in: 12th AIAA/ISSMO Multidisciplinary Analysis and Optimization Conference, Multidisciplinary Analysis Optimization Conferences, American Institute of Aeronautics and Astronautics, Victoria, British Columbia, Canada, 2008. doi: $10.2514 / 6.2008-5877$.

[2] R. M. Ajaj, M. I. Friswell, D. Smith, A. T. Isikveren, A conceptual wingbox weight estimation model for transport aircraft, The Aeronautical Journal 117 (1191) (2013) 533-551. doi:10.1017/S0001924000008174.

URL http://dx.doi.org/10.1017/S0001924000008174

[3] G. Bindolino, G. Ghiringhelli, S. Ricci, M. Terraneo, Multilevel Structural Optimization for Preliminary Wing-Box Weight Estimation, Journal of Aircraft 47 (2) (2010) 475-489. doi:10.2514/1.41552.

URL http://dx.doi.org/10.2514/1.41552

[4] M. C. Chambers, M. D. Ardema, A. P. Patron, A. S. Hahn, H. Miura, M. D. Moore, Analytical Fuselage and Wing Weight Estimation of Transport Aircraft, Technical Memorandum 110392, NASA Ames Research Center, Moffett Field, California, USA (4 1996).

URL https://ntrs.nasa.gov/archive/nasa/casi.ntrs.nasa.gov/ $19960025262 \cdot p d f$

[5] A. Elham, G. L. Rocca, M. van Tooren, Development and implementation of an advanced, design-sensitive method for wing weight estimation, Aerospace Science and Technology 29 (1) (2013) 100-113. doi:10.1016/j.ast.2013.01.012. URL http://www.sciencedirect.com/science/article/pii/ S1270963813000291

[6] S. A. Andrews, R. E. Perez, D. Wowk, Wing weight model for conceptual design of nonplanar configurations, Aerospace Science and Technology 43 (2015) 51-62. doi:10.1016/j.ast.2015.02.011. 
URL

http://www.sciencedirect.com/science/article/pii/ S1270963815000681

[7] P. O. Jemitola, G. Monterzino, J. Fielding, Wing mass estimation algorithm for medium range box wing aircraft, The Aeronautical Journal 117 (1189) (2013) 329-340. doi:10.1017/S0001924000008022.

[8] M. Kaufman, V. Balabanov, A. A. Giunta, B. Grossman, W. H. Mason, S. L. Burgee, R. T. Haftka, L. T. Watson, Variable-complexity response surface approximations for wing structural weight in hsct design, Computational Mechanics 18 (2) (1996) 112-126. doi:10.1007/BF00350530.

URL http://dx.doi.org/10.1007/BF00350530

[9] J. Frommer, W. Crossley, Enabling Continuous Optimization for Sizing Morphing Aircraft Concepts, in: 43rd AIAA Aerospace Sciences Meeting and Exhibit, Aerospace Sciences Meetings, American Institute of Aeronautics and Astronautics, Reno, Nevada, USA, 2005. doi:10.2514/6.2005-816.

URL http://dx.doi.org/10.2514/6.2005-816

[10] M. Skillen, W. Crossley, Developing Morphing Wing Weight Predictors with Emphasis on the Actuating Mechanism, in: 47th AIAA/ASME/ASCE/AHS/ ASC Structures, Structural Dynamics, and Materials Conference, Structures, Structural Dynamics, and Materials and Co-located Conferences, American Institute of Aeronautics and Astronautics, Newport, Rhode Island, USA, 2006. doi:10.2514/6.2006-2042.

URL http://dx.doi.org/10.2514/6.2006-2042

[11] M. D. Skillen, W. A. Crossley, Modeling and Optimization for Morphing Wing Concept Generation II. Part 1; Morphing Wing Modeling and Structural Sizing Techniques, Tech. Rep. NASA/CR-2008-214902/PT1, NASA Langley Research Center, Hampton, Verginia, USA (2 2008).

URL http://hdl .handle.net/2060/20080009777

[12] P. F. Albuquerque, P. V. Gamboa, M. A. Silvestre, Mission-Based Multidisciplinary Aircraft Design Optimization Methodology Tailored for Adaptive Tech- 
nologies, Journal of Aircraft (2017) 1-16doi:10.2514/1.C034403.

URL https://doi.org/10.2514/1.C034403

[13] D. Sousa, P. V. Gamboa, D. Melo, Aerodynamic performance of aerofoils obtained from a geometric offset applied to a given initial aerofoil, Open Engineering 6 (2016) 98. doi:10.1515/eng-2016-0098.

[14] P. Santos, J. Sousa, P. Gamboa, Variable-span wing development for improved flight performance, Journal of Intelligent Material Systems and Structures 28 (8) (2017) 961-978. doi:10.1177/1045389X15595719.

URL https://doi.org/10.1177/1045389X15595719

[15] ANSYS, Mechanical APDL and Mechanical Applications Theory Reference, Tech. Rep. Release 13.0, ANSYS, Inc., Canonsburg, PA, United States of America (11 2010).

[16] ANSYS, Mechanical APDL Structural Analysis Guide, Tech. Rep. Release 15.0, ANSYS, Inc., Canonsburg, PA, United States of America (11 2013).

[17] Y. Zhao, S. A. Billings, H. Wei, P. G. Sarrigiannis, Tracking time-varying causality and directionality of information flow using an error reduction ratio test with applications to electroencephalography data., Physical review. E, Statistical, nonlinear, and soft matter physics 86 (5 Pt 1) (2012) 51919. doi:10.1103/PhysRevE.86.051919.

[18] J. R. Taylor, Covariance and Correlation, University Science Books, 1997, pp. 209-266.

URL http://www . uscibooks. com/taylornb.htm

[19] Certification Specifications for Very Light Aeroplanes CS-VLA, Amendment 1, European Aviation Safety Agency (3 2009).

[20] E. Torenbeek, The Wing Structure and Its Weight, John Wiley \& Sons, 2013, pp. 319-362. doi:10.1002/9781118568101.ch11.

URL http://dx.doi.org/10.1002/9781118568101.ch11

[21] J. D. Anderson Jr., Fundamentals of Aerodynamics, 5th Edition, McGraw-Hill Education, 2010. 
[22] M. Drela, XFOIL 6.94 User Guide, Tech. rep., Massachusetts Institute of Technology \& Astro Harold Youngren Aerocraft, Inc. (12 2001).

[23] Van Dijk Pultrusion Products, DPP high performance micro-pultrusion profiles - Specification sheet (6 2014).

URL http://www.dpp-pultrusion.com/wp-content/uploads/2012/12/ DPP-specificationsheet-2014.pdf

[24] P. Santos, P. Gamboa, P. Santos, J. Silva, Structural Design of a Composite Variable-Span Wing, in: 4th International Conference on Integrity, Reliability and Failure, Funchal, 2013.

[25] P. Gamboa, P. Santos, J. Silva, P. Santos, Flutter Analysis of a Composite Variable-Span Wing, in: 4th International Conference on Integrity, Reliability and Failure, Funchal, 2013. 
2018-07-25

\section{Effect of design parameters on the mass}

of a variable-span morphing wing based

on finite element structural analysis and optimizatic

Santos, Pedro D. R.

Elsevier

Pedro D.R. Santos, Diogo B. Sousa, Pedro V. Gamboa and Yifan Zhao. Effect of design parameters on the mass of a variable-span morphing wing based on finite element structural analysis and optimization. Aerospace Science and Technology, Volume 80, Issue September, 2018, pp. 587-603

https://doi.org/10.1016/j.ast.2018.07.033

Downloaded from Cranfield Library Services E-Repository 